\title{
Perceived motion in structure from motion: Pointing responses to the axis of rotation
}

\author{
FRANK E. POLLICK \\ ATR Human Information Processing Research Laboratories, Kyoto, Japan \\ SHIN'YA NISHIDA \\ NTT Basic Research Laboratories, Tokyo, Japan \\ and \\ YASUHARU KOIKE and MITSUO KAWATO \\ ATR Human Information Processing Research Laboratories, Kyoto, Japan
}

\begin{abstract}
We investigated the ability to match finger orientation to the direction of the axis of rotation in structure-from-motion displays. Preliminary experiments verified that subjects could accurately use the index finger to report direction. The remainder of the experiments studied the perception of the axis of rotation from full rotations of a group of discrete points, the profiles of a rotating ellipsoid, and two views of a group of discrete points. Subjects' responses were analyzed by decomposing the pointing responses into their slant and tilt components. Overall, the results indicated that subjects were sensitive to both slant and tilt. However, when the axis of rotation was near the viewing direction, subjects had difficulty reporting tilt with profiles and two views and showed a large bias in their slant judgments with two views and full rotations. These results are not entirely consistent with theoretical predictions. The results, particularly for two views, suggest that additional constraints are used by humans in the recovery of structure from motion.
\end{abstract}

The ability to organize the changing retinal image into a reliable representation of the three-dimensional (3-D) world is an essential task for the visual system. Recent theoretical work has shown how to recover an object's 3-D shape from minimal amounts of its 2-D motion, and empirical work has examined the ability of these theories to explain human perception. Most of these theories and the related psychophysics have emphasized the recovery of 3-D structure, whereas less attention has been given to the closely related question of recovered 3-D motion. The recovery of 3-D motion is nonetheless theoretically fundamental to the recovery of structure from motion (SFM) and takes on different roles in different theories. One essential property of 3-D motion is the direction of the axis of rotation, and there are neurophysiological data to suggest that neurons in the posterior parietal cortex are capable of encoding the direction of the axis of rotation (Sakata, Kusunoki, \& Tanaka, in press). The present ex-

\footnotetext{
We wish to thank K. Nakane, Y. Tohkura, and E. Yodogawa for their support of this research. In addition, we thank Jeff Liter, Mike Braunstein, Jim Todd, John Andersen, and Michael Landy for helpful comments on previous drafts. Portions of this work were performed at the ATR Auditory and Visual Perception Research Laboratories. Correspondence should be addressed to Frank E. Pollick, ATR Human Information Processing Research Laboratories, 2-2 Hikaridai, Seika-cho, Sorakugun, Kyoto 619-02, Japan.
}

-Accepted by previous editor, Charles W. Eriksen periments investigated recovery of the direction of the axis of rotation from various SFM displays, such as rotating points and the profiles of rotating ellipsoids. (Profiles are also known as occluding contours, apparent contours, or outlines.)

The issue of 3-D motion recovery is treated differently among theories for the recovery of shape from minimal numbers of views (Bennett, Hoffman, Nicola, \& Prakash, 1989; Huang \& Lee, 1989; Koenderink \& Van Doorn, 1991; Todd \& Bressan, 1990; Ullman, 1979) and from the profiles of a smooth surface (Cipolla \& Blake, 1992; Giblin \& Weiss, 1987; Vaillant \& Faugeras, 1992). Some of these theories eliminate components of the 3-D motion and obtain an abstract representation of shape, other theories include all the motion information and simultaneously recover the 3-D shape and motion, yet others rely on knowledge about a component of 3-D motion (e.g., angular velocity, tilt or slant of the axis of rotation) to obtain the 3-D shape. These theoretical distinctions and their psychological implications for perceiving the direction of the axis of rotation are described first for the recovery of shape from minimal numbers of views and then for the recovery of shape from profiles.

The ability to recover 3-D motion and structure from orthographic projection is greatly influenced by the assumption of rigidity and the number of views permitted. From two views of four points and without assuming rigidity, but with the assumption that a 3-D linear transform relates the two views, it is possible to obtain the 
shape modulo an arbitrary affine transformation (Koenderink \& Van Doorn, 1991). The resulting structure is typically known as the affine structure (Todd \& Bressan, 1990). This recovery of affine structure is done at the expense of eliminating the component of motion specifying rotation about the line of sight (curl). Because the curl component has no information about the relative depth of points on an object (Koenderink, 1986), there is no loss of structural information. But, of course, removing the curl component alters the motion information. For example, without the curl component, it is impossible to know the slant of the axis of rotation. From two views of four points and with the assumption of rigidity, the 3-D Euclidean metric structure can be found up to a oneparameter family of solutions. The 3-D Euclidean metric family of solutions preserves all the motion information, but as shown by Bennett et al. (1989), the family of solutions are parameterized by the slant of the axis of rotation. Thus, the slant of the axis of rotation is known, but only up to a one-parameter family. The addition of a third view resolves the ambiguities obtained for two views, allowing recovery of a unique (unique up to a reflection ambiguity in slant) 3-D metric solution to the structure and direction of the axis of rotation (Ullman, 1979).

If subjects viewing SFM displays could use all the information that is theoretically available, then, from three or more views, they should be capable of recovering the 3-D metric structure. However, mixed results exist as to whether subjects indeed use the third view to obtain a unique structure and what happens with the addition of more views. The results of several studies have shown that perception of SFM "builds up" over more views (Eby, 1992; Hildreth, Grzywacz, Adelson, \& Inada, 1990), and other results have shown that performance with only two views is quite good and that there is no dramatic increase with more views (Braunstein, Hoffman, \& Pollick, 1990; Pollick \& Cheng, 1990; Todd \& Bressan, 1990). Thus, it might be that two views serve as the basis of human SFM perception. Consistent with this ability to recover structural properties from only two views, and the result that observers are insensitive to structural deformations arising from stretches along the line of sight (Norman \& Todd, 1993), is the suggestion that human SFM perception is affine in nature (Todd \& Bressan, 1990). However, alternative explanations exist for interpretations of these stretching displays (Pollick, 1993), and the issue is yet unresolved.

The profiles of a smooth solid object are sufficient to specify its 3-D surface geometry given that one fixes the solid object and knows the motion of the camera (Cipolla \& Blake, 1992; Giblin \& Weiss, 1987; Vaillant \& Faugeras, 1992). Although it is straightforward to transform the problem to one of fixed camera and unknown object motion, the problem is how to then obtain properties of the motion, such as the angular velocity and the direction of the axis of rotation. This problem has been addressed (Giblin, Pollick, \& Rycroft, in press; Giblin, Rycroft, \& Pollick, 1992; Pollick, Giblin, Rycroft, \& Wilson, 1992); it has been shown that, from a set of profiles of a smooth object making a complete rotation, it is possible to recover the projection of the axis of rotation from the symmetry of the envelope of the profiles. Once the projection of the axis of rotation is recovered, then the tilt of the axis of rotation is known and several other properties follow. For example, finding the tilt makes it possible to find the relative depth of points on the profile, and if either the angular velocity or slant of the axis of rotation is known, then a unique 3-D structure can be recovered.

If the rotation is measured over some small interval of time, it is still possible to recover the tilt of the axis of rotation. This is done by finding the points on the profile that satisfy the frontier condition (Giblin et al., in press). This condition dictates that a point's vertical velocity divided by its horizontal position must remain constant (measured from an origin lying on the projection of the axis of rotation, with vertical being along the direction of the projected axis). This condition arises from the fact that every time a point on the profile has its (2-D) normal parallel to the projection of the axis of rotation, then its projected velocity in the vertical direction is identical to the projection of the true 3-D velocity. This is a sort of correspondence; however, because the points that "correspond" are continually changing, they contribute no information above what can be obtained with two views. This result underscores a theoretical similarity between profiles and two views: Even if the tilt of the axis is known, it is still impossible to find the slant from a minimum motion sequence.

For profiles, psychophysical research has primarily concentrated on the ability to perceive 3-D shape (Todd, 1985; Wallach \& O'Connell, 1953) and the inverse relationship between perceived shape of an object and its angular velocity (Cortese \& Andersen, 1991; Pollick, 1993). The perception of the axis of rotation has been studied by Norman and Todd (in press) in the context of a rigidity discrimination task where subjects viewed the profiles of two rotating ellipsoids and were asked to discriminate whether the pair was rotating about the same axis of rotation (rigid) or rotating about different axes of rotation (nonrigid). They found that the discrimination greatly improved if the profiles of the two ellipsoids intersected.

The review of previous research shows that although the axis of rotation plays a central role in theories of SFM, the perception of the axis of rotation has not been extensively studied. The present set of experiments was designed to measure subjects' abilities to report the axis of rotation for a wide range of orientations. The subjects reported by orienting a finger in a direction identical to the direction of the perceived axis of rotation. Pointing was chosen because of the ease with which it could be used and the familiarity people have with indicating direction by pointing.

The present study consisted of a series of four experiments that was run twice. The first run investigated whether pointing judgments of the axis of rotation were correlated to ratings of perceived structure (Braunstein, 
1962; Dosher, Landy, \& Sperling, 1989; Green, 1961; Todd, Akerstrom, Reichel, \& Hayes, 1988). In addition, the pointing results were fit with a simple linear model and plotted taking into account the orthographic ambiguity inherent in the displays. The second run investigated more closely the role of bias and variability in subjects' responses, and the results were plotted collapsing across the orthographic ambiguity. In the series of four experiments, Experiment 1 of the series quantified subjects' abilities to orient the index finger to a static, real object (a metal bar). In Experiments 2-4, subjects oriented the finger to match the axis of rotation of computer-generated SFM displays containing different amounts of information to specify the axis of rotation.

\section{EXPERIMENT 1}

Experiment 1 investigated the subjects' ability to point a finger in the direction of a real physical object. It was performed in an unrestricted visual environment, allowing the use of any available cues to orientation. The purpose was, in a sense, to obtain calibration data for the finger-pointing system over the range of input that would be used in later experiments. The orientations studied were those contained in the upper right quadrant of the unit sphere, and responses were measured on the basis of the subject's pointing of the right index finger.

\section{Method}

Subjects. Six subjects were divided into two groups: an experienced group composed of 3 of the coauthors, and a naive group composed of 3 staff members of the lab who were naive to the purpose of the experiment.

Design. The independent variables were the slant and the tilt of a metal rod. The subjects responded once for each of 56 different orientations. Of the $\mathbf{5 6}$ orientations, 54 were spaced in a factorial fashion among 6 tilts $\left(0^{\circ}, 18^{\circ}, 36^{\circ}, 54^{\circ}, 72^{\circ}\right.$, and $\left.90^{\circ}\right)$ and 9 slants $\left(18^{\circ}, 36^{\circ}, 54^{\circ}, 72^{\circ}, 90^{\circ}, 108^{\circ}, 126^{\circ}, 144^{\circ}\right.$, and $\left.162^{\circ}\right)$, where slant is defined as the angle between the viewing direction and the axis of rotation and tilt is the angle between the horizontal and the projection of the axis of rotation onto the viewing plane. The remaining two orientations corresponded to $0^{\circ}$ and $180^{\circ}$ of slant, where tilt is undefined.

Stimuli and Apparatus. The stimuli consisted of an oriented $40 \times 2.5 \times 2.5 \mathrm{~cm}$ straight metal rod attached to a robot arm (Mitsubishi Movemaster, RV-M2) under the control of a microcomputer (NEC 9801). The 56 orientations were achieved by moving only the base and wrist of the robot so that the attached end of the metal rod was always in approximately the same location at a height of $100 \mathrm{~cm}$. The subject's eye position was approximately $160 \mathrm{~cm}$ from the robot and raised slightly higher than the metal rod so that the $180^{\circ}$ slant condition could be seen. In order to provide a uniform background, a large black screen was placed at $140 \mathrm{~cm}$ from the rear of the robot.

The subjects' pointing responses were collected with an OPTOTRAK 3-D position-sensing system (Northern Digital), with accuracy of approximately $0.2 \mathrm{~mm}$. Four markers (infrared emitting diodes) were attached to a metal casing $(2.5 \times 2.5 \times 7.5 \mathrm{~cm})$ that was worn on the index finger of the subject's right hand. To facilitate visibility of the markers, one pair was mounted flush to the side of the casing and another pair was mounted at $45^{\circ}$ facing forward. No measurements were taken unless one of the pairs of markers was visible to the OPTOTRAK cameras, which were located directly to the left of the seated subject.

Procedure. The subjects were instructed to direct the right index finger in a direction identical to that of the metal rod. The pointing was constrained so that the tip of the finger acted as if it was the attached end of the metal rod (direction was measured from the tip of the finger toward the knuckle). The subject was allowed to freely move his/her head and body, provided that he/she remained seated. Once the subject was confident that the index finger was in the correct position and had indicated this to the experimenter, a 1-sec recording was made of the finger position at a rate of $50 \mathrm{~Hz}$. The 50 samples were averaged to obtain $x, y$, and $z$ locations for each marker. Each subject performed a single session, seeing the 56 orientations in a randomized order and responding by pointing. At the end of the session, the subjects were asked to point in the directions (measured from the tip of the finger to the knuckle) right $(x)$, up $(y)$, backward $(z)$, and forward $(-z)$. This was done in order to assess the subjects' ability to align their personal coordinate systems with that used by the robot and OPTOTRAK.

\section{Results and Discussion}

The results were analyzed in two ways. The first analysis looked at the angular difference between the direction of the metal rod and the direction of the pointed finger. The second analysis broke the pointed directions into their component slant and tilt directions and looked at the relationship between actual slant and responded slant, as well as the relationship between actual tilt and responded tilt. This first technique provided an idea of the absolute accuracy, whereas the second was intended to find any bias in the responses.

The angular difference between the actual and recovered directions was termed the error angle. Figure la shows the error angles collapsed across tilts and plotted as a function of slant of the metal rod. The average errors were $8.8^{\circ}(S D=3.9)$ and $9.2^{\circ}(S D=3.7)$ for the experienced and the naive subjects, respectively. It can be seen that the lowest errors were for $0^{\circ}$ and $180^{\circ}$ slant; for the slants between $90^{\circ}$ and $172^{\circ}$, there was a substantial increase in error angle. However, this increase was probably due to the increased mechanical difficulty involved in pointing as the direction became closer to $180^{\circ}$. (For these directions, the subjects were required to point toward themselves with the tip of the finger.)

The results of decomposing the pointed direction into its components of slant and tilt are shown in Figures $1 \mathrm{~b}$ and $1 \mathrm{c}$. The plot of the pointed slant versus the presented slant is shown in Figure 1b. It can be seen that the regressions for both the experienced and the naive subjects were quite good, with $98 \%$ of the variability in responses accounted for by presented slant. Similar results were found for plots of pointed tilt versus presented tilt (Figure $1 \mathrm{c}$ ), where, for both naive and experienced subjects, 95\% of the variability was accounted for. Because the subsequent SFM experiments showed a substantial effect of slant on judgments of tilt, the tilt results of Figure 1c were broken down into individual regressions at each level of slant (slants greater than $90^{\circ}$ were reflected back), and Table 1 shows the results. It can be seen that as the slant 


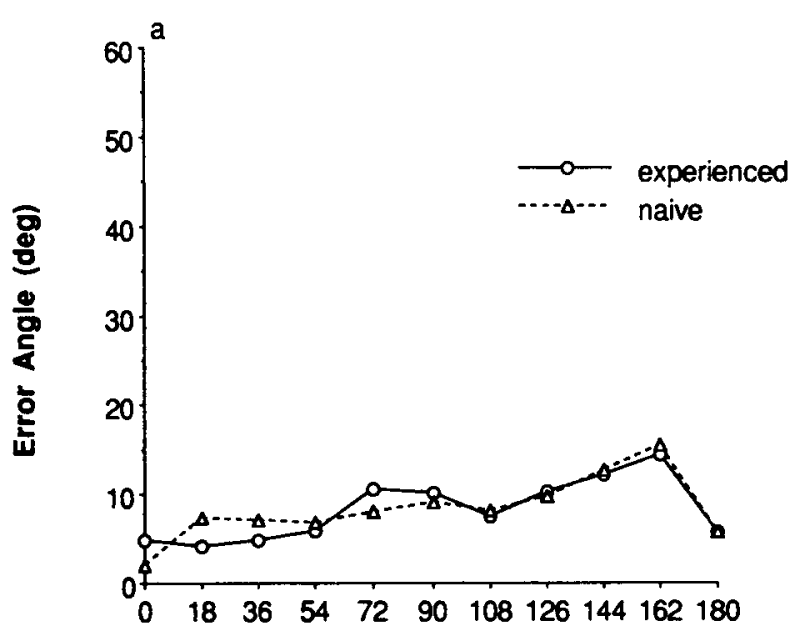

Slant (deg)

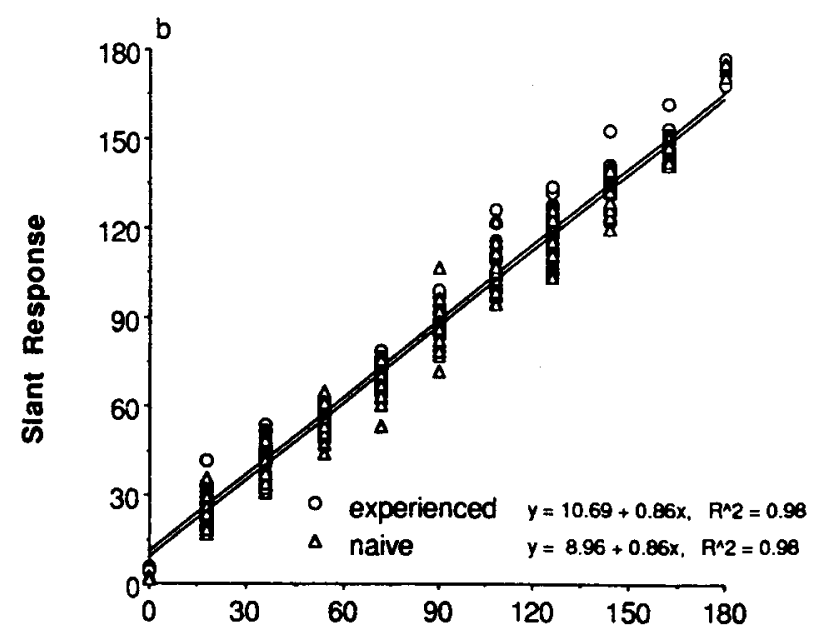

Slant (deg)

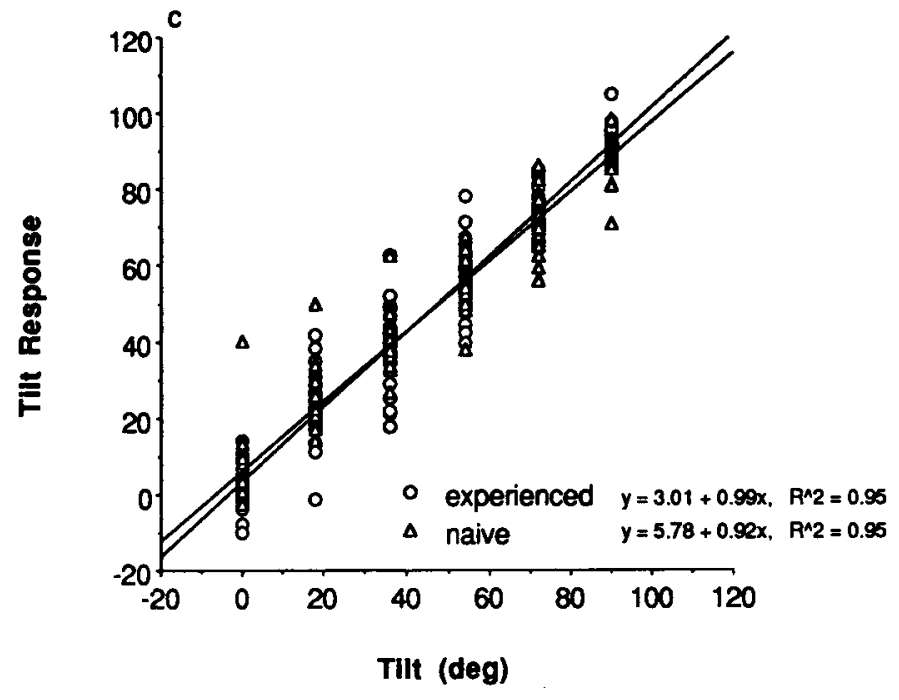

Figure 1. Results of pointing the direction of a static real object (Experiment 1), plotted for both naive and experienced subjects: (a) the error angle versus the slant of the axis of rotation, (b) data and regression lines for responded slant versus actual slant, and (c) data and regression lines for responded tilt versus actual tilt.

approached the viewing direction, the fits for the tilt regressions became worse. Some of this effect on tilt judgments could be expected on computational grounds because, for slants close to the viewing direction, deviations in finger position produce larger errors in tilt.

The final judgments of the coordinate directions demonstrated that the subjects were fairly accurate in aligning their coordinate systems with that of the external frame of reference used to make the measurements. The subjects' mean angular errors in pointing the $x, y, z$, and $-z$ directions were $2.0^{\circ}, 2.9^{\circ}, 3.8^{\circ}$, and $4.9^{\circ}$, respectively $(S D \mathrm{~s}=0.78,2.3,1.2$, and 2.5$)$. Since the $-\mathrm{z}$ direction corresponds to the direction of pointing to oneself, this larger error is consistent with the idea that some subjects had a bit more difficulty pointing in this direction.
The results of Experiment 1 indicated that both the naive and the experienced subjects could reliably match their finger orientation to the direction of a stimulus over a wide range of orientations. The average error angle of $9^{\circ}$, although not negligible, is small when one considers the large range of possible responses. The plots of responded slant and tilt versus presented slant and tilt showed that the subjects' pointing responses were closely tracking the changing direction of the stimulus.

\section{EXPERIMENT 2}

In Experiment 2, the subjects viewed displays simulating the motion of several points making full rotations about a fixed axis. These displays allowed several strategies 
Table 1

Regression Values for Experiment 1

\begin{tabular}{|c|c|c|c|}
\hline Slant (deg) & Slope & $y$ Intercept & $r^{2}$ \\
\hline \multicolumn{4}{|c|}{ Experienced Subjects } \\
\hline 18 & 0.98 & 4.25 & .90 \\
\hline 36 & 1.02 & 2.26 & .93 \\
\hline 54 & 0.98 & 3.52 & .97 \\
\hline 72 & 0.97 & 2.72 & .99 \\
\hline 90 & 0.97 & 1.60 & 1.00 \\
\hline \multicolumn{4}{|c|}{ Naive Subjects } \\
\hline 18 & 0.83 & 11.33 & .84 \\
\hline 36 & 0.93 & 5.68 & .97 \\
\hline 54 & 0.94 & 3.64 & .99 \\
\hline 72 & 0.95 & 4.12 & .98 \\
\hline 90 & 0.96 & 2.45 & .99 \\
\hline
\end{tabular}

from which to recover the axis of rotation. For example, the direction of the axis could be recovered by applying any of various SFM algorithms to the motions of points over a limited motion. The axis of rotation could also be recovered by tracing out the elliptical path of a single point over a complete rotation. (For fixed-axis rotation, every point traces out a circular path in a plane perpendicular to the axis of rotation; the projection of this circle to the image plane is an ellipse whose orientation gives the tilt of the axis and whose eccentricity gives the slant.) These techniques could also be combined in various ways to obtain a consistent estimate of the axis of rotation. Thus, the results obtained in Experiment 2 could be considered as an upper bound of performance with SFM stimuli.

\section{Method}

Subjects. The experienced group remained the same 3 subjects, but 1 of the 3 subjects in the naive group was switched to another staff member who was also unaware of the purpose of the experiment.

Design. The independent variables were the simulated slant and tilt of the axis of rotation of an SFM display. There were 31 possible axes of rotation; of these, 30 were arranged in a factorial fashion among 6 tilts $\left(0^{\circ}, 18^{\circ}, 36^{\circ}, 54^{\circ}, 72^{\circ}\right.$, and $\left.90^{\circ}\right)$ and 5 slants $\left(18^{\circ}\right.$, $36^{\circ}, 54^{\circ}, 72^{\circ}$, and $\left.90^{\circ}\right)$. The remaining orientation corresponded to $0^{\circ}$ slant, where tilt is undefined. It should be noted that due to the orthographic reflection ambiguity for slant, the number of possible direction-of-the-axis-of-rotation judgments available to the subjects was 56 [i.e., $(2 \times 31)-6]$.

Stimuli and Apparatus. A stimulus simulated the orthographic projection of 21 white points against a black background making complete rotations about a common axis of rotation. The simulated motion was continuous, with the $360^{\circ}$ divided into 200 frames. Each frame had an exposure duration of $30 \mathrm{msec}$. The initial point positions were chosen randomly from within the volume of the unit sphere.

The subjects were seated in a dimly lit room approximately $142 \mathrm{~cm}$ from the display screen of a Masscomp 5800 computer $(1,152 \times 910$ resolution). The subject's head was in a chinrest. The displays were viewed monocularly. The field of view of the display screen was restricted to $6^{\circ}$ of visual arc by an aperture located $36 \mathrm{~cm}$ in front of the chinrest. This gap of $36 \mathrm{~cm}$ allowed the subject enough room to comfortably adjust the position of the finger within view.

The arrangement for measuring the finger position was identical to that used in the previous experiment.
Procedure. Each subject participated in a single session of 62 trials divided into two blocks of 31 trials. There was a short rest in the middle of each block and between blocks. Both blocks contained a randomized order of the complete set of displays. The first block was used as practice to develop experience with the rating scales, and thus only the second block was used in subsequent analysis. For each trial, the subject was instructed to position the right index finger in a direction identical to that of the axis of rotation (henceforth referred to as pointing the direction of the axis of rotation). The stimuli rotated indefinitely; when the subject was satisfied with the position of the finger, he/she indicated this to the experimenter and a $1-\mathrm{sec}$ interval at $50 \mathrm{~Hz}$ was recorded.

Immediately following the measurement of finger position, the subjects gave judgments of the direction of rotation. These judgments were same/different responses to an arrow attached to the metal casing worn on the finger. The purpose of the judgments was to help verify which of the orthographic interpretations was being perceived.

After the judgments of the direction of rotation were made, two more judgments were made to rate the nature of the perceived structure. The purpose of these judgments was to find any possible relationship between subjective perception of the structure and performance on judging the axis of rotation. The first judgment rated the depth of the object on a scale from 1 to 5 (with 5 being the maximum). The second judgment rated the rigidity of the object, where 5 corresponded to the object always being seen as rigid and 1 corresponded to the object always being perceived as undergoing a nonrigid deformation.

\section{Results and Discussion}

The data were analyzed similarly to those of Experiment 1 . The angular difference between the direction of the axis of rotation and the direction of the pointed finger was computed. In addition, the pointed directions were reduced to their component slant and tilt directions, and the regression between real and simulated directions was analyzed.

The error-angle results are shown in Figure 2a collapsed across tilts and plotted as a function of simulated slant of the axis of rotation. The average errors were $15.1^{\circ}$ $(S D=5.2)$ and $33.7^{\circ}(S D=17.3)$ for the experienced and the naive subjects, respectively. The naive subjects showed particularly large errors for slants less than $90^{\circ}$, whereas the experienced subjects showed much greater accuracy and did not report any reflection interpretations, responding that all axes of rotation had $90^{\circ}$ of slant or less.

The results of decomposing the pointed direction into its components of slant and tilt are shown in Figures $2 \mathrm{~b}$ and $2 c$, respectively. The plot of the pointed slant versus the displayed slant is shown in Figure $2 b$. It can be seen that the regressions for the experienced subjects were better than those for the naive subjects. The naive subjects showed a strong tendency to overestimate slant for small values, resulting in a large $y$ intercept. Similar trends were found for plots of pointed tilt versus displayed tilt (Figure $2 \mathrm{c}$ ), where the experienced subjects performed substantially better than the naive ones. The breakdown of tilt results into individual regressions at each level of slant (Table 2) shows that the experienced subjects could adequately report the tilt of the axis of rotation out to the smallest level of slant, but the performance of naive subjects decreased dramatically for values less than $72^{\circ}$ of slant. 

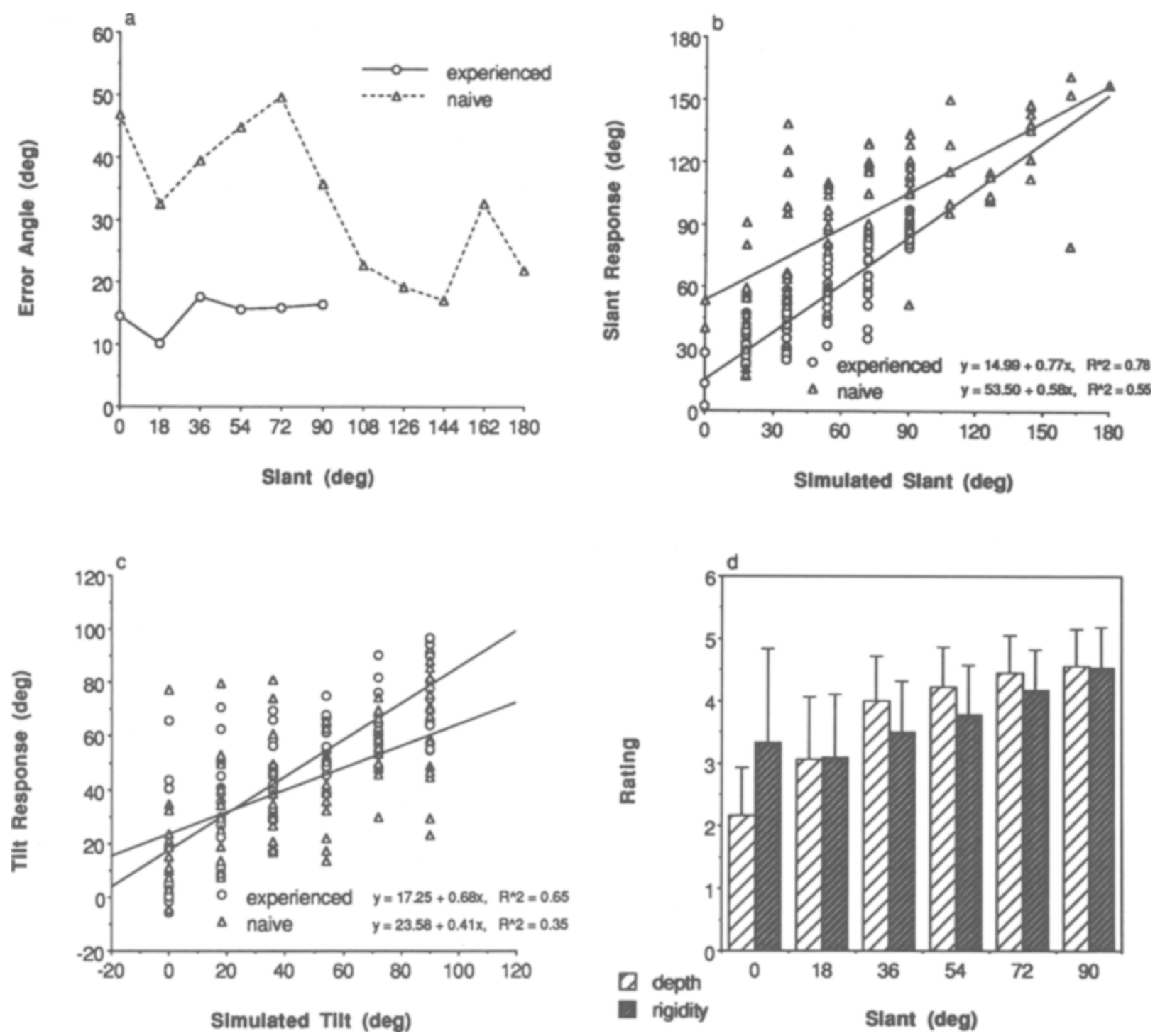

Figure 2. Results of pointing the direction of the axis of rotation from 21 points making full rotations (Experiment 2), plotted for both naive and experienced subjects: (a) the error angle versus the simulated slant of the axis of rotation, (b) data and regression lines for responded slant versus simulated slant, (c) data and regression lines for responded tilt versus simulated tilt, and (d) mean values of the depth and rigidity ratings plotted versus simulated slant (error bars indicate standard deviations).

The rating data were analyzed with two goals in mind: (1) to find any relationship between the subjective ratings of structure and the orientation of the axis of rotation, and (2) to find any relationship between the subjective rating of structure and the performance at pointing the direction of the axis of rotation.

To find the relationship between the ratings and the orientation of the simulated axis, analyses of variance (ANOVAs) were performed on both the depth and the rigidity ratings to test for effects of tilt, slant, and their interaction. The ANOVAs were performed using the 6 tilts and 5 slants, excluding the axis direction with undefined tilt. The ANOVA on the depth judgments yielded a significant effect of slant $[F(4,20)=12.9, p<.01]$, with judgments of depth decreasing as the axis of rotation ap- proached the viewing direction. A similar result was found for the ANOVA on the rigidity ratings: slant had a significant effect $[F(4,20)=8.7, p<.01]$, with judgments of rigidity decreasing as the axis of rotation approached the viewing direction. Plots of the mean ratings versus slant are shown in Figure 2d.

The relationship between subjective ratings of structure and errors in pointing was studied by calculating the Spearman rank correlation coefficients between the ratings and the absolute value of the errors in tilt and slant. The values used to calculate these correlation coefficients were the pooled responses of the 6 subjects for the cases when tilt was defined $(n=180)$. The depth ratings were found to be negatively correlated to the error in pointing tilt $\left(r_{\mathrm{S}}=\right.$ $-.15, p<.05)$. The rigidity ratings were found to be 
Table 2

Regression Values for Experiment 2

\begin{tabular}{cccc}
\hline Slant $(\mathrm{deg})$ & \multicolumn{1}{c}{ Slope } & $y$ Intercept & $r^{2}$ \\
\hline \multicolumn{4}{c}{ Experienced } \\
18 & 0.54 & 25.62 & \\
36 & 0.63 & 24.91 & .47 \\
54 & 0.76 & 15.19 & .52 \\
72 & 0.70 & 16.45 & .80 \\
90 & 0.79 & 4.10 & .73 \\
& Naive Subjects & .89 \\
18 & 0.14 & 41.62 & \\
36 & 0.33 & 27.27 & .05 \\
54 & 0.29 & 22.57 & .24 \\
72 & 0.62 & 15.49 & .76 \\
90 & 0.67 & 10.97 & .74 \\
\hline
\end{tabular}

negatively correlated to error in both pointing slant $\left(r_{\mathrm{S}}=\right.$ $-.17, p<.05)$ and pointing tilt $\left(r_{\mathrm{S}}=-.17, p<.05\right)$.

The results of Experiment 2 showed that overall, the subjects could point the direction of the axis of rotation. Compared with the real static object, there was a large increase in the average error angle, particularly for the naive subjects. However, both groups showed similar trends on the plots of pointed versus simulated slant and tilt. The primary difference between groups was that the experienced subjects were better able to report the tilt at low values of slant. The rating data showed that ratings of rigidity and depth decreased as the simulated axis of rotation approached the viewing direction. This finding is similar to that obtained by Loomis and Eby (1988) in their study of the contribution of the curl component of motion to the perception of depth in SFM. The ratings of depth and rigidity were also found to have a slight but statistically significant negative correlation with errors in judging slant and tilt.

\section{EXPERIMENT 3}

In Experiment 3, the subjects viewed the profiles of ellipsoids of revolution making complete rotations. With respect to information content, these displays were similar to those of Experiment 2 in that complete rotations provided sufficient information to recover the axis of rotation and small extents of rotation specified the tilt of the axis but not its slant. (From complete rotations, the tilt of the axis is given by the symmetry of the envelope of the family of profiles; using the period of rotation to estimate the angular velocity allows recovery of the slant. For small extents of rotation, by identifying frontier points, one can find the tilt of the axis but not its slant; Giblin et al., 1993). Thus, for profiles of smooth objects, the axis of rotation was specified exactly by a complete rotation but was ambiguous for any small portion of the rotation.

\section{Method}

The 6 subjects who participated in Experiment 2 also participated in Experiment 3. Profiles of rotating ellipsoids were used as stimuli. The design and procedure were the same as those used in Experiment 2.
Stimuli. A stimulus consisted of the profiles of an ellipsoid of revolution (ratio of major to minor axis $=3.0$ ) making complete rotations about a fixed axis. The simulated motion appeared continuous with the $360^{\circ}$ divided into 54 frames, each frame with an exposure duration of $107 \mathrm{msec}$. Each profile was depicted as a solid black form moving in front of a background of bright dots; motion was simulated by appropriately turning the background points off and on (Cortese \& Andersen, 1991). The maximum extent of the simulated object was $3.5^{\circ}$ of visual arc. The background was composed of $50 \%$ of the $1,152 \times 910$ pixels randomly switched on. In order to eliminate effects of the orientation of the ellipsoid relative to the axis of rotation, the orientation of the ellipsoid was randomized across trials. For the profiles of an ellipsoid, the center of the profile is the projection of the center point of the ellipsoid and thus can be thought of as a point of correspondence. For this reason, the axis was penetrated through the center of the ellipsoid so the center point would have no motion.

\section{Results and Discussion}

The data were analyzed in the same way as was done with the data of Experiment 2 . The error-angle results are shown in Figure 3a collapsed across tilts and plotted as a function of simulated slant. The average errors were $22.1^{\circ}(S D=12.5)$ and $39.7^{\circ}(S D=24.4)$ for the experienced and the naive subjects, respectively.

The results of decomposing the pointed direction into its components of slant and tilt are shown in Figures $3 \mathrm{~b}$ and $3 c$, respectively. The plots of the pointed slant versus the displayed slant are shown in Figure $3 \mathrm{~b}$. The regressions for both the experienced subjects and the naive subjects accounted for much of the variability, with the experienced subjects performing better. Quite different results were found for plots of pointed tilt versus displayed tilt (Figure 3c), where neither experienced nor naive subjects' regressions accounted for much of the variance. However, the analysis of tilt regressions at individual levels of slant (Table 3 ) shows that tilt was recovered for slants approaching $90^{\circ}$.

The relationship between the subjective ratings of structure and the orientation of the simulated axis was studied in the same way as was done for Experiment 2. The ANOVA on the depth ratings yielded a significant effect of slant $[F(4,20)=10.2, p<.01]$, with judgments of less depth when the axis of rotation was near the viewing direction. The ANOVA on the rigidity ratings yielded no significant effects. The rating values as a function of slant are shown in Figure 3d.

The relationship between subjective ratings of structure and errors in pointing was studied, just as in Experiment 2, by calculating the Spearman correlation coefficients from the pooled data of the 6 subjects for the cases when tilt was defined $(n=180)$. The depth ratings were found to be negatively correlated to the errors in pointing slant $\left(r_{\mathrm{S}}=-.15, p<.05\right)$ and tilt $\left(r_{\mathrm{S}}=-.17, p<\right.$ $.05)$. The rigidity ratings were not found to be correlated to error in either slant or tilt.

The results of Experiment 3 showed that both the naive and the experienced subjects had the ability to indicate the orientation of the axis of rotation under certain conditions. Both groups showed similar trends; however, the 


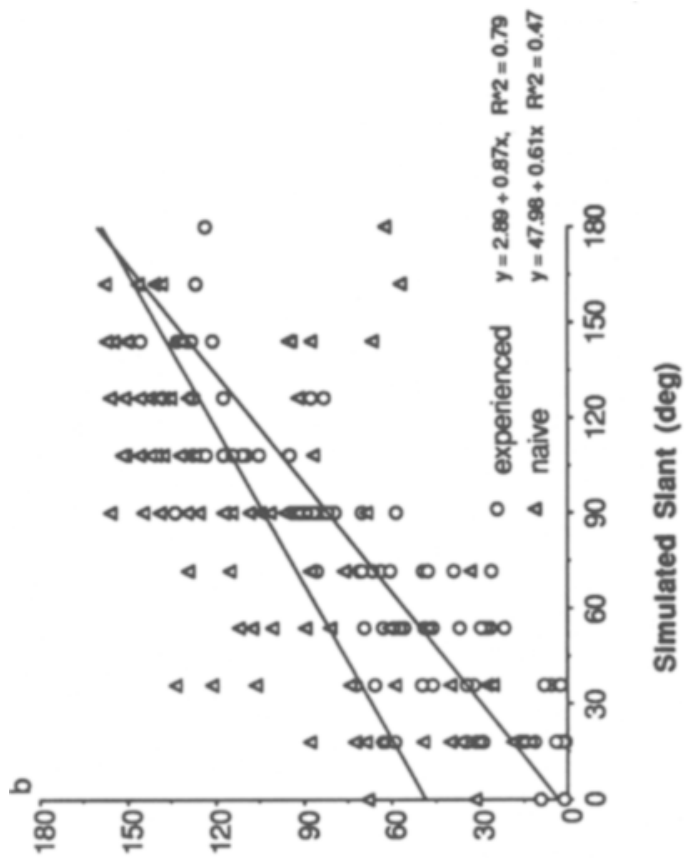

(Bep) esuodsey zuв/S

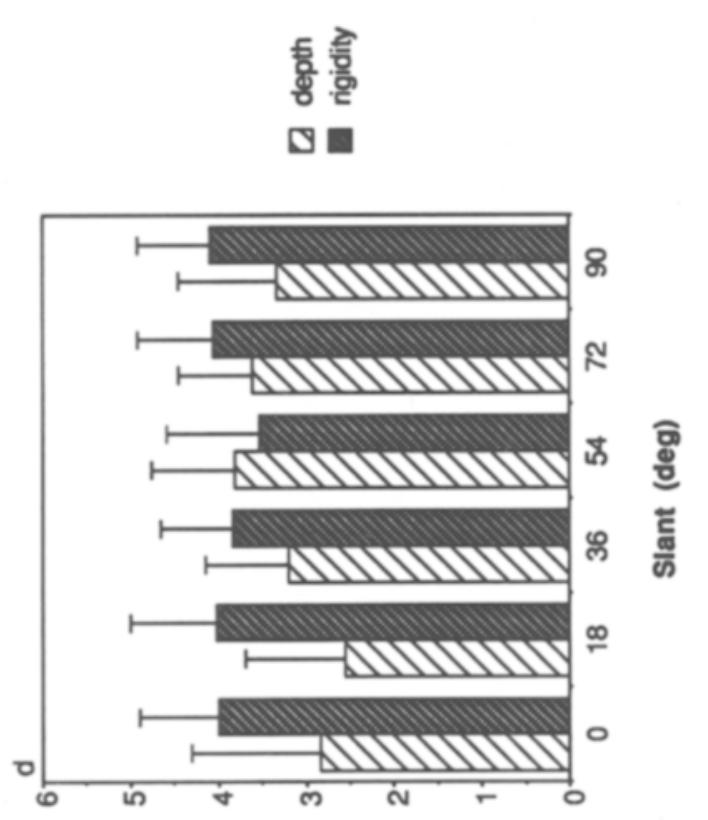

Bupey

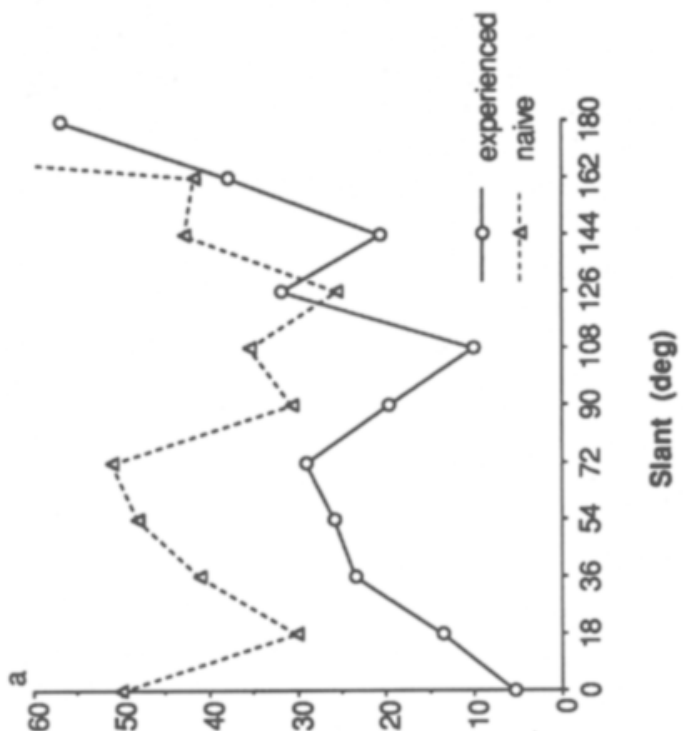

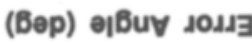

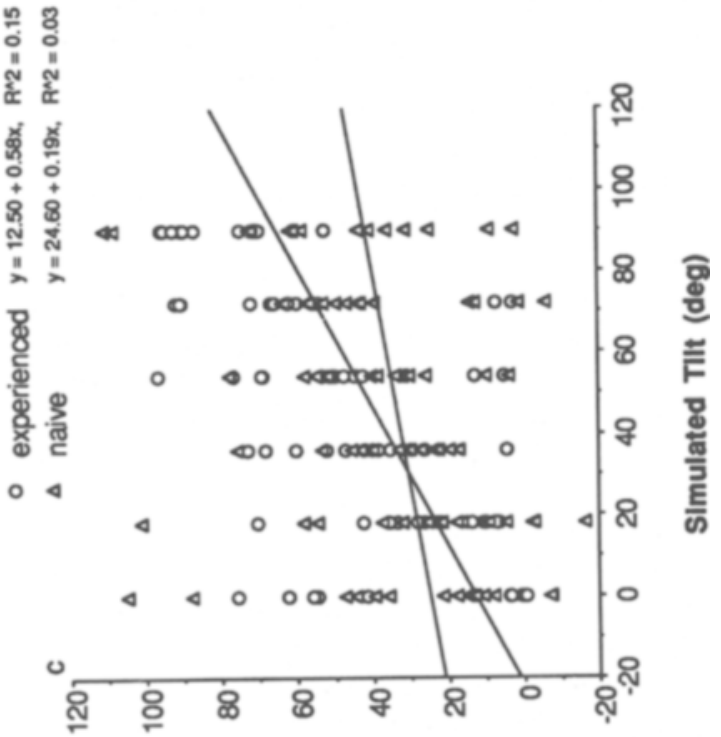

(Bep) esuodsey йL
을 
Table 3

Regression Values for Experiment 3

\begin{tabular}{|c|c|c|c|}
\hline Slant (deg) & Slope & $y$ Intercept & $r^{2}$ \\
\hline \multicolumn{4}{|c|}{ Experienced Subjects } \\
\hline $\begin{array}{l}18 \\
36 \\
54 \\
72 \\
90\end{array}$ & $\begin{array}{l}0.16 \\
0.66 \\
0.48 \\
0.74 \\
0.87\end{array}$ & $\begin{array}{r}25.65 \\
-9.34 \\
26.83 \\
12.38 \\
7.00\end{array}$ & $\begin{array}{l}.01 \\
.12 \\
.31 \\
.74 \\
.92\end{array}$ \\
\hline \multicolumn{4}{|c|}{ Naive Subjects } \\
\hline $\begin{array}{l}18 \\
36 \\
54 \\
72 \\
90\end{array}$ & $\begin{array}{l}0.01 \\
0.08 \\
0.17 \\
0.08 \\
0.61\end{array}$ & $\begin{array}{r}41.06 \\
26.23 \\
22.05 \\
25.96 \\
7.69\end{array}$ & $\begin{array}{l}.00 \\
.00 \\
.05 \\
.01 \\
.48\end{array}$ \\
\hline
\end{tabular}

naive subjects' data showed greater variability, with less of the variance accounted for in the tilt and slant regressions. The subjects could identify the tilt of the axis of rotation when the axis was perpendicular to the viewing direction, but this ability broke down as the axis approached the viewing direction. During debriefing, some of the subjects commented that the task was made more difficult by a high rate of orthographic reversals. This could have been one of the factors contributing to the variability in the data. The rating data again showed that depth ratings decreased as the axis of rotation approached the viewing direction and that errors in judging both slant and tilt had a slight but statistically significant negative correlation with depth ratings.

\section{EXPERIMENT 4}

In Experiment 4, the subjects observed two views of seven points rotating about a fixed axis. For these twoview orthographic projections, unlike for the multiview displays used in Experiments 1 and 2, it is theoretically impossible to recover the slant of the axis of rotation. It is still possible, however, to recover the tilt of the axis of rotation.

\section{Method}

The 6 subjects who participated in Experiments 2 and 3 also participated in Experiment 4. Two views of seven points were used as stimuli. The design and procedure were the same as those used in Experiment 2.

Stimuli. A stimulus consisted of two views of seven points rotating through an angular displacement of $11^{\circ}$. The pattern of the displayed rotation was to show Frame 1 and then Frame 2, each with an exposure duration of $466 \mathrm{msec}$. Following Frame 2, the screen went dark for $932 \mathrm{msec}$, and then the sequence began again at Frame 1. This timing allowed meaningful judgments of the direction of rotation. The points were chosen randomly from the volume of a unit sphere, and their motion was constrained. The first constraint forced each point to move at least $5 \%$ of the radius of the unit sphere between frames. This was done to prevent motionless points that would have been cues to the location of the axis of rotation. The second constraint enforced nearest neighbor matching between frames; each point in Frame 1 had to be closer to the 2-D position of itself in Frame 2 than to the position of any other point in Frame 2. This was done to assure correct correspondence matching.

\section{Results and Discussion}

The data were analyzed in the same way as was done with the data of Experiments 2 and 3. The error angle results are shown in Figure 4a. The average errors were $22.6^{\circ}(S D=11.1)$ and $35.5^{\circ}(S D=14.8)$ for the experienced and naive subjects, respectively.

The results of decomposing the pointed direction into its components of slant and tilt are shown in Figures $4 \mathrm{~b}$ and $4 \mathrm{c}$, respectively. The plots of the pointed slant versus the simulated slant are shown in Figure $4 \mathrm{~b}$. The regressions for both the experienced subjects and the naive subjects accounted for much of the variability, with the experienced subjects performing better. Quite different results were found for plots of pointed tilt versus displayed tilt (Figure 4c) where neither experienced nor naive subjects' regressions accounted for much of the variance. However, the analysis of tilt regressions at individual levels of slant (Table 4) shows that it was possible to recover the tilt of the axis for slants approaching $90^{\circ}$. For the experienced subjects, the regressions improved as the axis approached the frontoparallel plane, but only for slants of $90^{\circ}$ and $72^{\circ}$ did the regressions account for much of the variance. The naive subjects seemed to be able to judge the tilt only when the slant was $90^{\circ}$.

The relationship between the subjective ratings of structure and the orientation of the simulated axis was studied in the same way as was done in Experiments 2 and 3. The ANOVA on the depth judgments yielded no significant effects. The ANOVA on the rigidity ratings yielded a significant effect of slant $[F(4,20)=5.3, p<.01]$, with judgments of greater rigidity when the axis of rotation was close to the viewing direction. The ratings of depth and rigidity as a function of slant are shown in Figure 4d.

The relationship between subjective ratings of structure and errors in pointing was studied. The depth ratings were found to be negatively correlated to the error in judging tilt $\left(r_{\mathrm{S}}=-.20, p<.05\right)$, but no other correlations were significant.

The results of Experiment 4 for two views were quite similar to those obtained in Experiment 3 for profiles. Both naive and experienced subjects showed substantial error angles. They could indicate the slant of the axis of rotation with some accuracy but could indicate the tilt of the axis accurately only when the slant was close to $90^{\circ}$ (perpendicular to the viewing direction). Consistent with the two previous SFM experiments, the rating data showed decreasing depth ratings as the axis of rotation approached the viewing direction and also a slight but statistically significant negative correlation between errors in pointing tilt and ratings of depth.

\section{CUMULATIVE RESULTS OF EXPERIMENTS 1-4}

It is instructive to compare the performance at pointing the direction of the axis of rotation across experiments and combine the results of the naive and experienced 


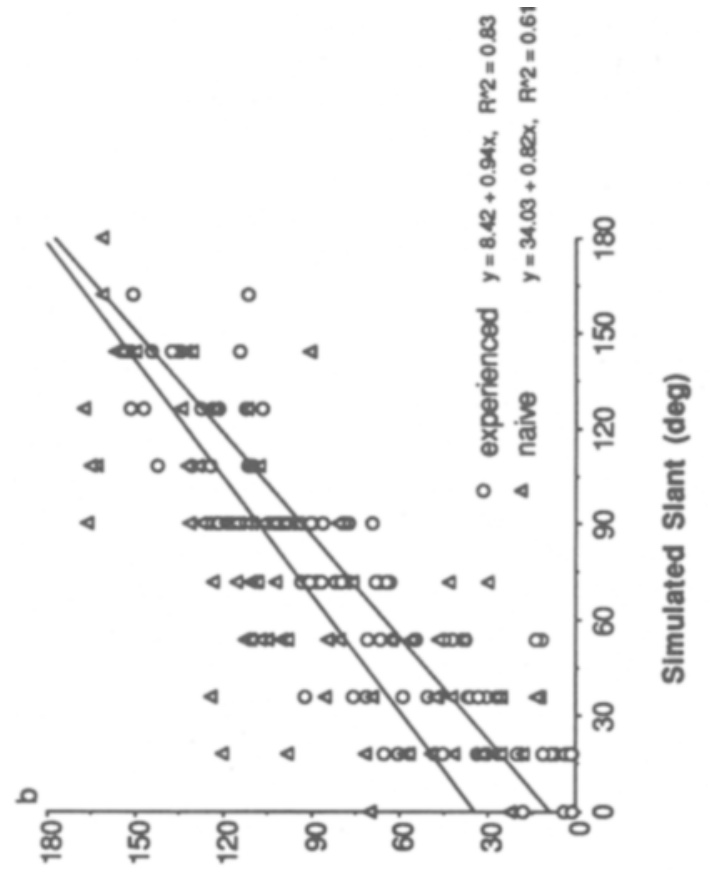

(Bep) esuodsey jueIS

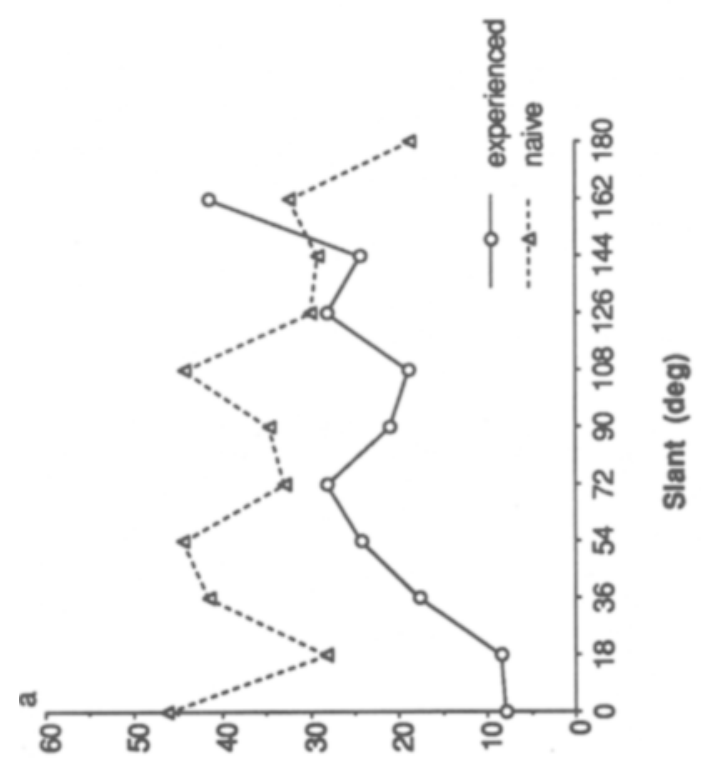

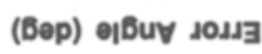

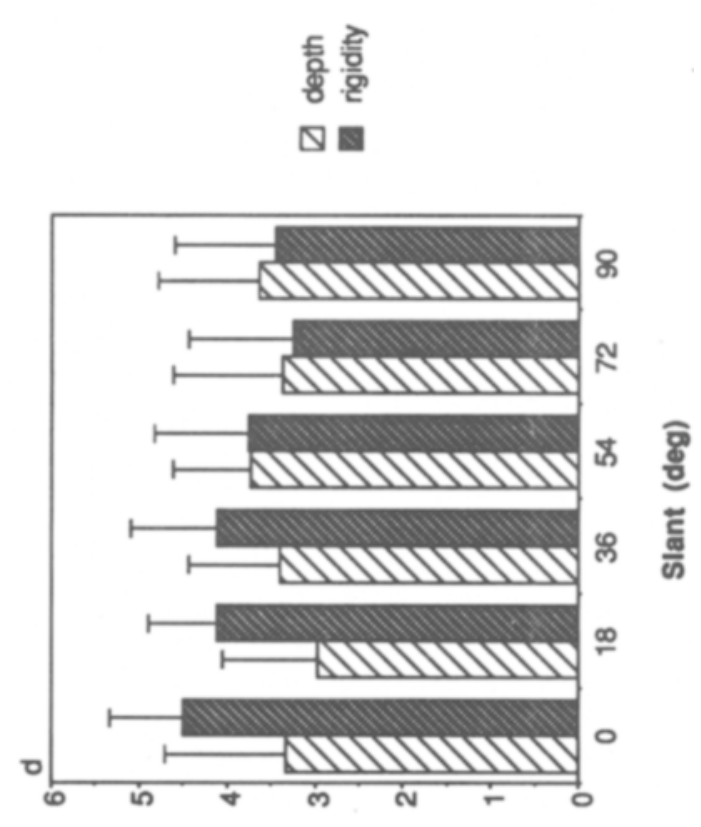

Bupвy

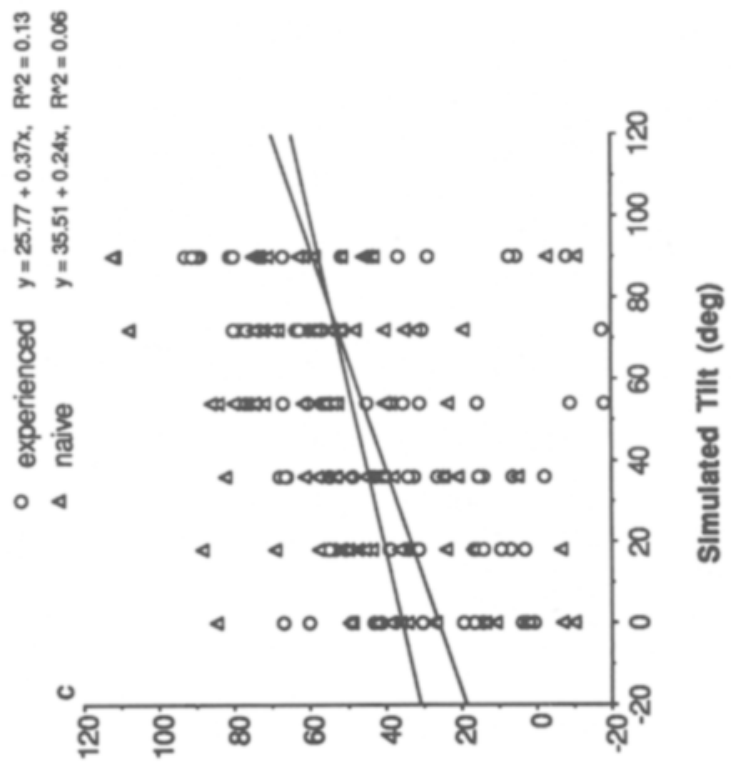

(Bep) esuodsey 
Table 4

Regression Values for Experiment 4

\begin{tabular}{|c|c|c|c|}
\hline Slant (deg) & Slope & $y$ Intercept & $r^{2}$ \\
\hline \multicolumn{4}{|c|}{ Experienced Subjects } \\
\hline 18 & -0.28 & 48.27 & .03 \\
\hline 36 & 0.08 & 37.65 & .01 \\
\hline 54 & 0.29 & 29.50 & .18 \\
\hline 72 & 0.89 & 5.95 & .79 \\
\hline 90 & 0.85 & 7.47 & .89 \\
\hline \multicolumn{4}{|c|}{ Naive Subjects } \\
\hline 18 & -0.19 & 55.60 & .02 \\
\hline 36 & 0.21 & 35.13 & .05 \\
\hline 54 & 0.35 & 37.57 & .19 \\
\hline 72 & 0.06 & 36.71 & .01 \\
\hline 90 & 0.77 & 12.55 & .71 \\
\hline
\end{tabular}

groups. These cumulative results are shown in Figure 5, where it can be seen that subjects performed best at matching the orientation of the real object and that performance decreased for SFM displays but was relatively consistent across the various types of SFM displays.

The absolute accuracy of the pointing response, indicated by the error angles (Figure 5a), shows that, relative to performance on matching a real object (average angular error $9^{\circ}$ ), performance decreased markedly for reporting the axis of rotation of SFM displays (average angular error $28^{\circ}$ ). For the SFM displays, performance was best at a slant of $90^{\circ}$ (axis of rotation perpendicular to the viewing direction).

The result of decomposing the pointing response into slant and tilt components (Figures $5 \mathrm{~b}$ and $5 \mathrm{c}$ ) shows that performance was quite good for the real object and decreased for SFM displays. Comparing results between the three SFM displays showed that slant recovery was consistent across all the types of SFM displays, whereas tilt recovery was better for full rotations than for profiles and two views. For profiles and two views, tilt could be recovered only at a slant of $90^{\circ}$; however, for full rotations, tilt could be recovered at lower slants.

The subjective ratings of depth and rigidity showed that, for all the SFM displays, ratings of depth decreased as the axis approached the viewing direction. In addition, there was a slight but statistically significant negative correlation between ratings of depth and errors in pointing tilt. Overall, the decreases in ratings of depth and rigidity did not seem sufficient to fully account for the changes in pointing performance. Thus, it is unlikely that the errors in pointing the direction of the axis of rotation were simply the result of subjects' failing to perceive a 3-D object. However, the negative correlation between ratings of depth and errors in pointing tilt is consistent with what one would predict of methods that need the correct tilt to recover the 3-D structure.

\section{EXPERIMENTS 5-8}

In general, the regression results of Experiments 1-4 showed that, frequently, much of the variability in subjects' responses could be accounted for by the simple linear model, suggesting that subjects' pointing responses were sensitive to the changes being simulated. However, for some conditions, the error was considerable; therefore, additional experiments were performed to examine whether the error resulted from variability of the responses or response bias. This was accomplished by obtaining multiple responses to each axis of rotation over a similar set of stimuli and experiments.

\section{Method}

The apparatus used in Experiments 5-8 was identical to that used in Experiments 1-4. For 10 repetitions of each stimulus condition, the subjects pointed the direction of the axis of rotation and gave judgments of the direction of rotation (or in the case of the real object, simply matched directions of the object). The range of stimuli was identical to that used in the previous experiments but slightly less sampled. For the experiment involving judgments of the orientation of the metal bar, the slants were $0^{\circ}, 22.5^{\circ}, 45^{\circ}, 67.5^{\circ}, 90^{\circ}$, $112.5^{\circ}, 135^{\circ}, 157.5^{\circ}$, and $180^{\circ}$ and the tilts were $0^{\circ}, 30^{\circ}, 60^{\circ}$, and $90^{\circ}$. For the SFM experiments, the slants were (ignoring the orthographic ambiguity) $0^{\circ}, 22.5^{\circ}, 45^{\circ}, 67.5^{\circ}$, and $90^{\circ}$ and the tilts were $0^{\circ}, 30^{\circ}, 60^{\circ}$, and $90^{\circ}$.

Two subjects (the first author, F.E.P., and a naive subject, T.O.) ran the entire sequence of four experiments. Additional data were obtained from Naive Subject H.K. for judgments of the metal bar and from Naive Subject Y.I. on the three SFM experiments.

The experiment for matching orientation to the metal bar was blocked into five sessions of 60 trials that took place over 2 days. The SFM experiments were divided into three sessions of 51,68 , and 51 trials. For each session, the order of trials was randomized and, at the conclusion of each session, the subjects pointed in the directions of $x, y, z$, and $-z$ to verify that they were calibrated to the coordinate system used by the measuring device.

Data processing was similar to that of the previous four experiments. However, one notable exception was the treatment of the perception of orthographic reversals in the SFM displays. In Experiments 1-4, the axis was taken to be its responded orientation (either toward or away from the subject); however, in the present series, the responses that were away from the subject were reflected back, as if to be toward the subject. This was done for two reasons: (1) it facilitated the data analysis to have equal numbers of responses for each condition; and (2) for the SFM experiments involving discrete points, the percentage of "away" responses was negligible $(0 \%$ for full rotation and $3 \%$ for two views). A reversal rate of $44 \%$ was obtained for the profile displays, but analysis yielded similar results whether or not the responses were reflected back.

\section{Results and Discussion Experiments 5-8}

To check that the results were consistent with those of Experiments 1-4, we first calculated the regressions and average error angles. The error-angle results showed average errors of 4.9 for the static object and 23.5 for the SFM displays-slightly lower than that obtained before. The regressions (Table 5) showed a pattern of results similar to that obtained before; performance was best for the static object and worst for the tilt regressions of profiles and two views.

After we confirmed that the results were consistent with those obtained in the first series of experiments, the bias and variability of the responses were calculated. Bias was defined as the angular distance from the displayed slant (tilt) to the slant (tilt) of the centroid of the responses. Variability was defined as the average (unsigned) distance from the centroid to the individual responses. The fol- 

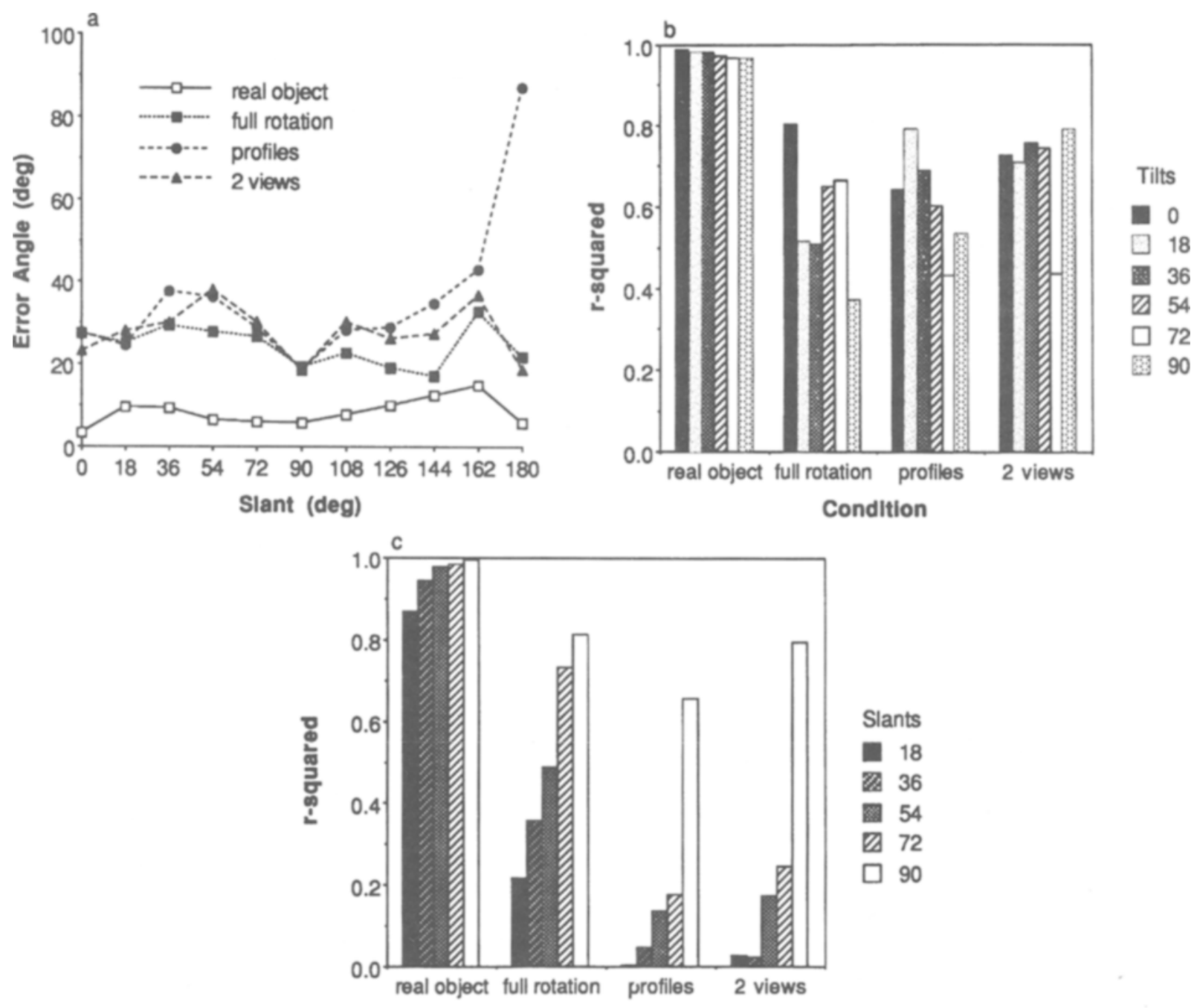

Condition

Figure 5. Cumulative results for all experiments averaged across all subjects: (a) the error angle versus the slant of the axis of rotation, (b) values of $r^{2}$ for the regressions of responded slant versus simulated slant shown for individual levels of tilt, and (c) values of $r^{2}$ for the regressions of responded tilt versus simulated tilt shown for individual levels of slant.

lowing paragraphs first describe the mean bias and variability (averaged across conditions) for individual subjects and then show how this bias and variability (averaged across subjects) were distributed across the individual levels of slant and tilt.

Figure 6 shows the bias and variability (averaged across conditions) for the individual subjects. It can be seen that, in many aspects, the performance of all subjects was roughly similar in magnitude and trends. For all subjects, the variability in both slant and tilt was highest for profiles and two views, decreased for full rotations, and was smallest for the real object. All subjects also showed the greatest amount of bias for full rotations and two views. Another similarity between subjects was that, in 11 of 12 cases, the variability in tilt was larger than the bias in tilt, and a similar trend existed for the slant judgments with

Table 5

Summary of Regression Values for Experiments 5-8

\begin{tabular}{clccccccc}
\hline & & \multicolumn{3}{c}{ Slant } & & \multicolumn{3}{c}{ Tilt } \\
\cline { 3 - 4 } \cline { 6 - 8 } Experiment & Condition & Slope & $y$ intercept & $r^{2}$ & & Slope & $y$ intercept & $r^{2}$ \\
\hline 5 & Static object & 0.86 & 13.96 & .97 & 0.93 & 4.22 & .96 \\
6 & Full rotation & 0.72 & 32.50 & .64 & 0.72 & 12.02 & .71 \\
7 & Profiles & 0.91 & 8.25 & .62 & 0.51 & 18.18 & .22 \\
8 & Two views & 0.75 & 24.39 & .60 & 0.51 & 23.00 & .355 \\
\hline
\end{tabular}



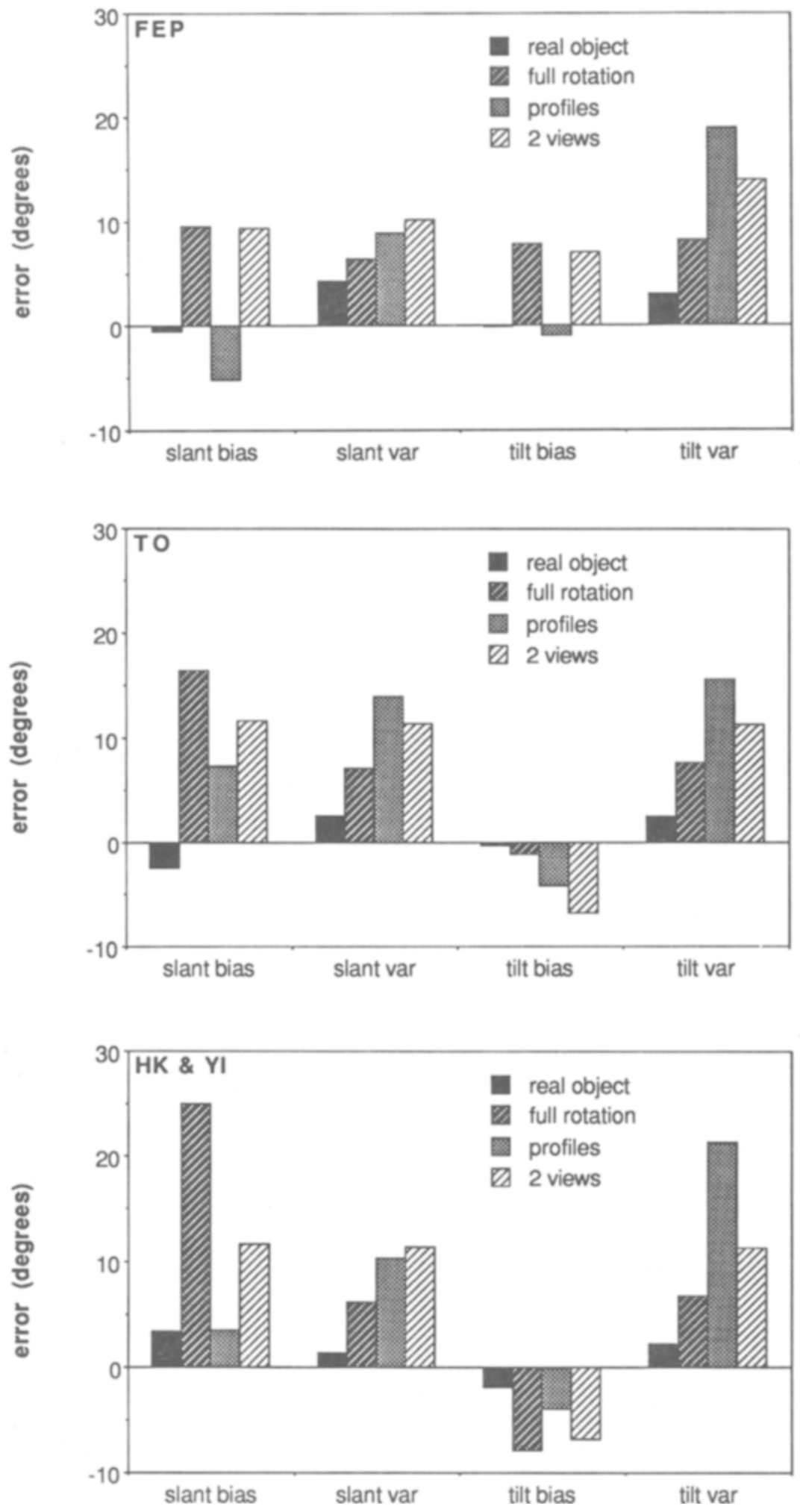

Figure 6. Average bias and variability of the tilt and slant judgments, plotted for individual subjects in Experiments 5-8. 
the exception of full rotations. This individual-subject data give some feel for the consistency among the different subjects and the trends across experiments, but it is most informative to view the bias and variability at the individual levels of slant and tilt.

The trends in variability and bias of both the slant and the tilt components of the responses can be seen in Figure 7. The significance of these trends was tested by calculating ANOVAs using the mean responses of individual subjects (see Table 6). Examining the trends of the tilt judgments, it can be seen that tilt was overestimated at small tilts and underestimated at large tilts. Also, there

a

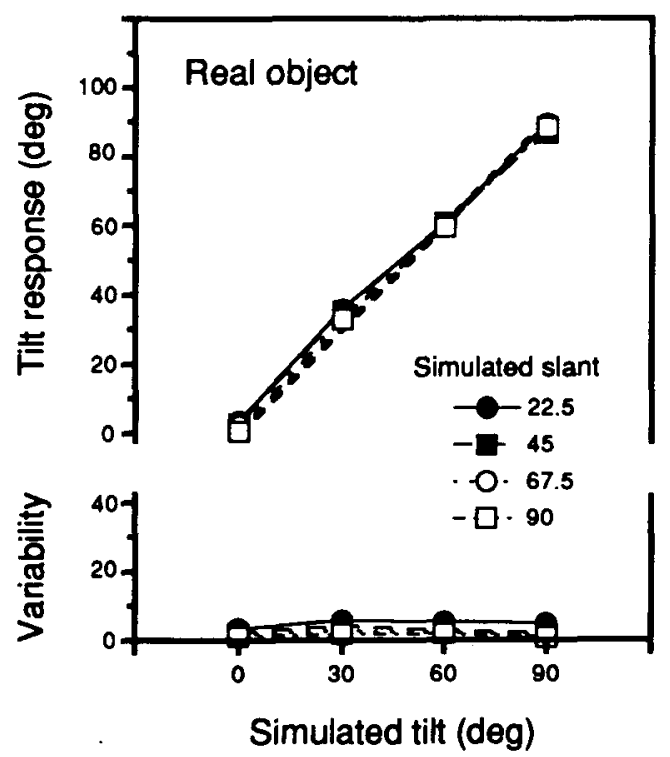

C
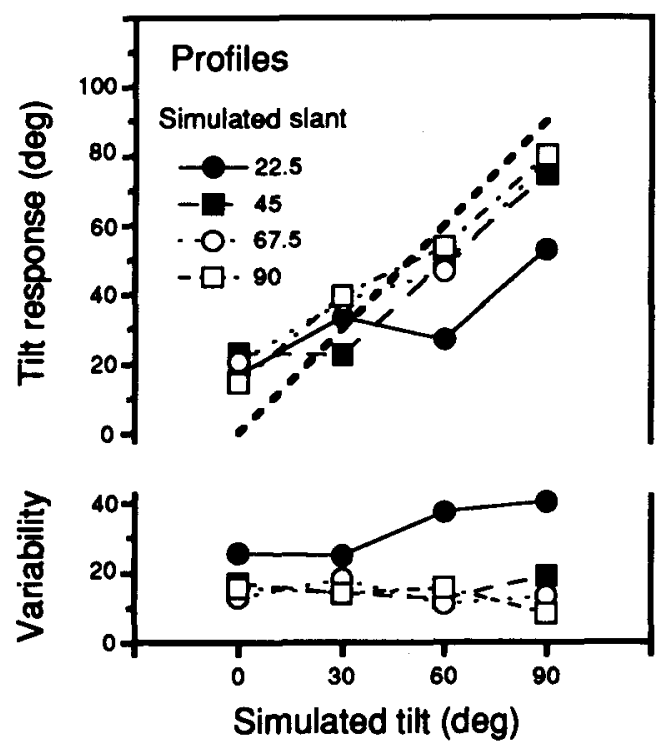

was an interaction between slant and tilt. However, it is likely that the bias in tilt judgments is best described as a regression toward the mean of the responses. Evidence for this comes from the fact that tilt bias seemed to covary with tilt variability, and this is most clearly seen for profiles and two views at a slant of $22.5^{\circ}$ (Figures $7 \mathrm{c}$ and 7d). (It should be noted, though, that for the SFM displays the ANOVA results showed no effects of tilt variability.) Examining the trends of the slant judgments, it can be seen that slant was overestimated at small slants and that this bias in slant judgments did not coincide with any changes in the variability of the slant judgments. In addition, there

b

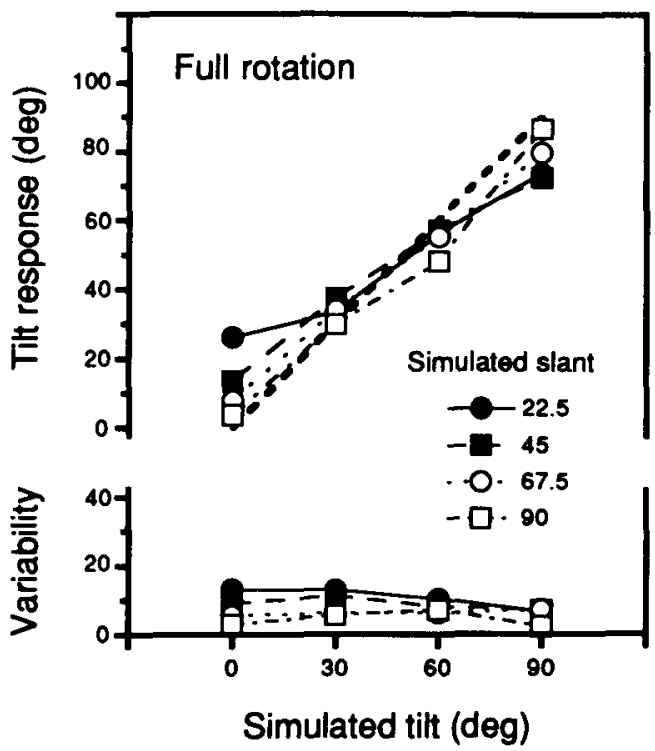

d

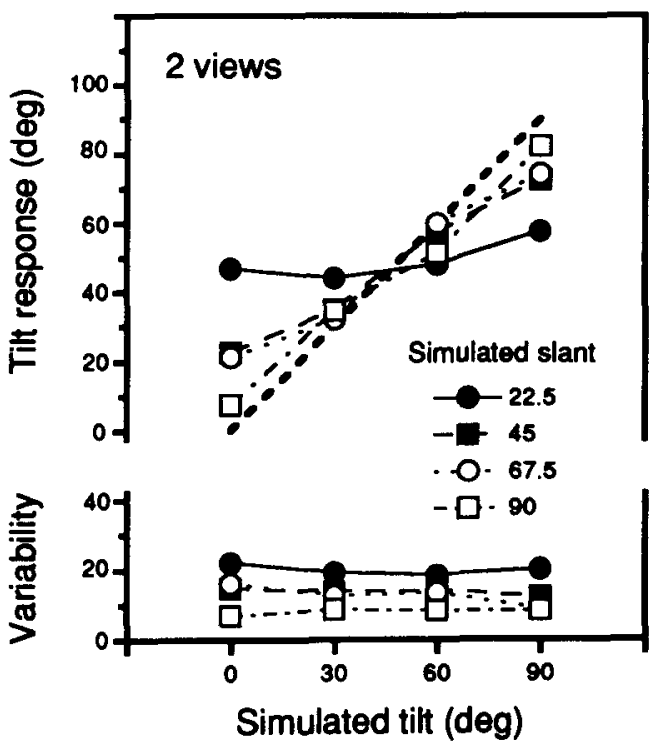

Figure 7 (above and facing page). Bias and variability of the tilt and slant judgments, averaged across subjects and plotted at the individual levels of slant and tilt. Plots a-d give the results for tilt in Experiments 5-8, and Plots e-h give the results for slant in Experiments 5-8. Bias is evident as the distance from the heavy dashed diagonal line to the response. 
$\theta$

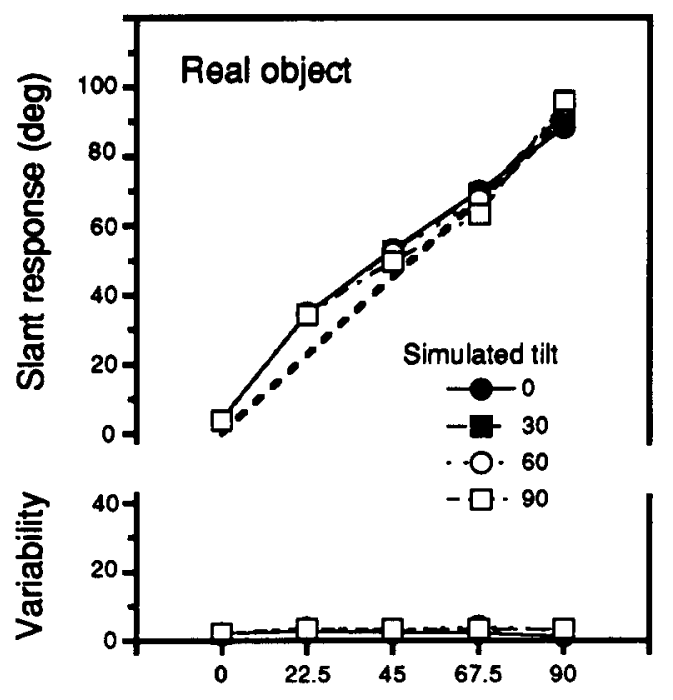

Simulated slant (deg)

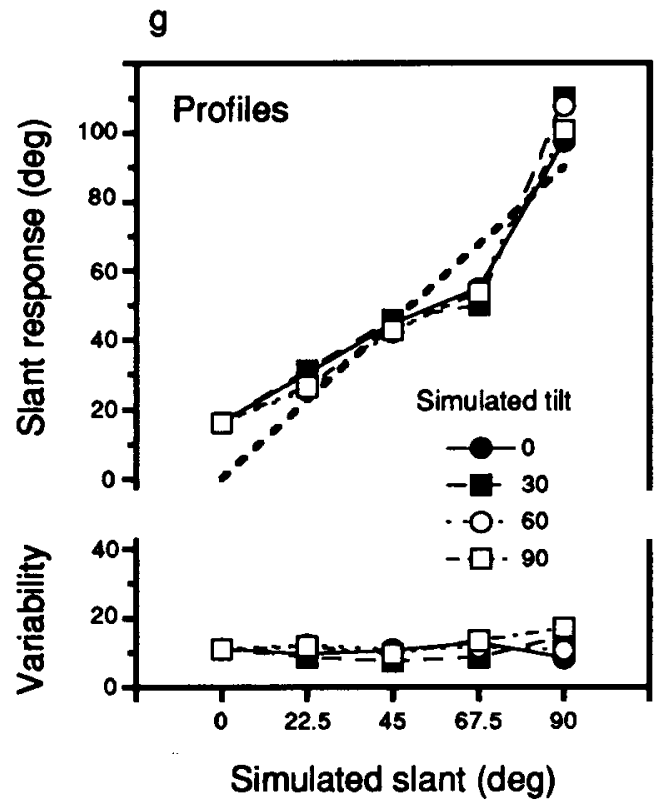

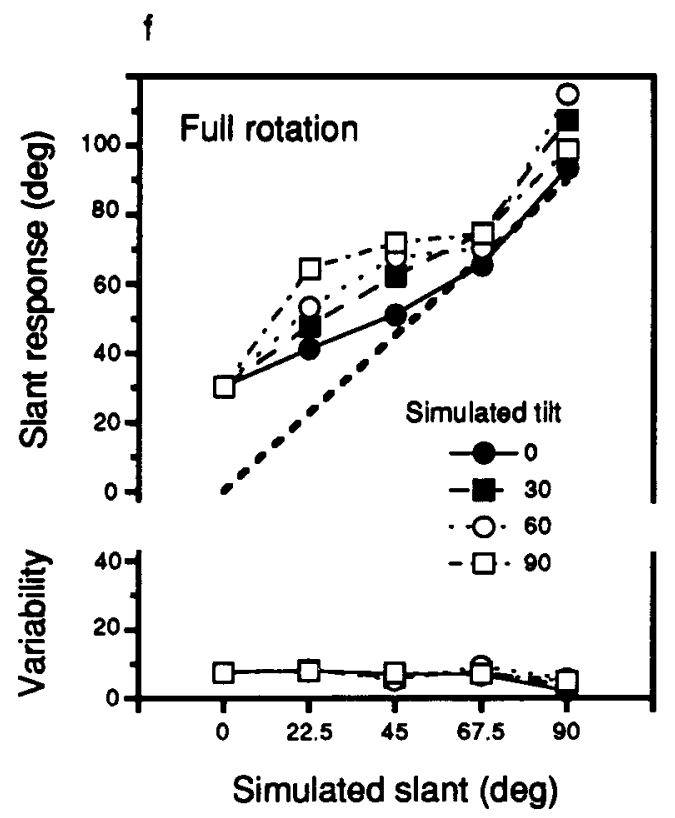

h

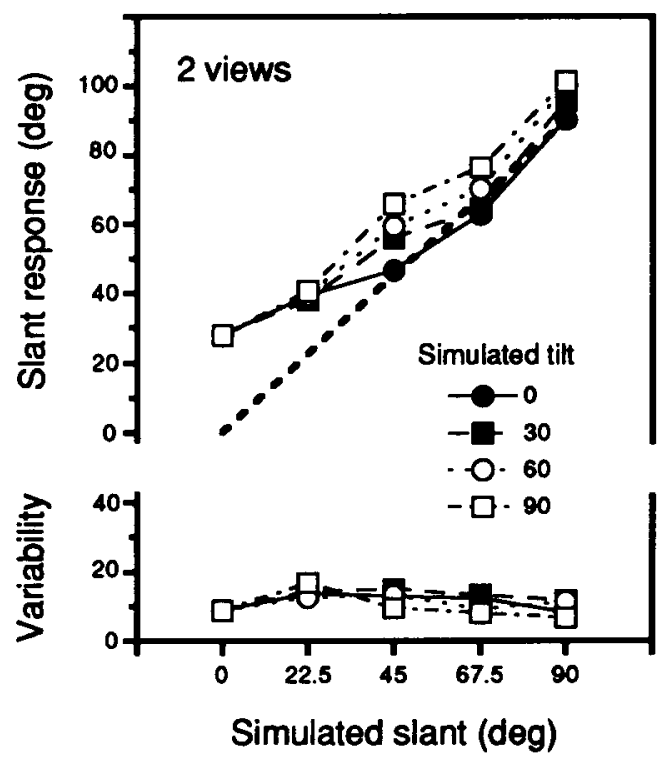

Figure 7 (continued).

was sometimes (most noticeably for full rotations and two views) an effect of tilt on slant bias, with judgments of slant becoming worse as tilt approached $90^{\circ}$.

Summarizing the results of bias and variability in the slant and tilt judgments: the bias of the tilt judgments seemed due to the tendency of the tilt responses to regress toward the mean, particularly at low slants. However, there seems to be no similar artifact, such as regression toward the mean, that might account for the bias found in the slant judgments. Thus, it seems likely that the large bias at small slants and the effect of tilt on slant bias were due to a misperception of the orientation of the axis of rotation.
Since there were, at times, large amounts of bias and variability of the responses, it was necessary to examine how much of this could have come from measurements of the pointing response itself. To answer this, we examined the responses given at the end of the experiments indicating the cardinal directions (each subject judged the $x, y, z$, and $-z$ directions at the completion of each session). The results of these judgments are given in Table 7 . It can be seen that the standard deviations of both slant and tilt were small, indicating that, among the sessions, individual subjects were able to consistently orient their fingers and align their personal coordinate systems with that of the measurement system. However, there was a 
Table 6

ANOVA Results for Slant and Tilt Bias from Experiments 5-8

\begin{tabular}{|c|c|c|c|c|c|}
\hline & Variable & Slant Bias & Slant Variability & Tilt Bias & Tilt Variability \\
\hline \multicolumn{6}{|c|}{ Experiment 5} \\
\hline \multirow[t]{3}{*}{ Real object } & slant & $F(6,12)=45.0^{*}$ & n.s. & n.s. & $F(6,12)=13.6^{*}$ \\
\hline & tilt & $F(3,6)=11.1^{*}$ & $F(3,6)=7.3 \dagger$ & $F(3,6)=14.2^{*}$ & $F(3,6)=13.3^{*}$ \\
\hline & slant $\times$ tilt & $F(18,36)=2.4 \dagger$ & n.s. & $F(18,36)=7.1^{*}$ & $F(18,36)=3.3^{*}$ \\
\hline \multicolumn{6}{|c|}{ Experiment 6} \\
\hline \multirow[t]{3}{*}{ Full rotations } & slant & $F(3,6)=21.7^{*}$ & n.s. & n.s. & n.s. \\
\hline & tilt & $F(3,6)=25.5$ & n.s. & $F(3,6)=5.4 \dagger$ & n.s. \\
\hline & slant $\times$ tilt & $F(9,18)=2.6 \dagger$ & n.s. & $F(9,18)=5.4^{*}$ & n.s. \\
\hline \multicolumn{6}{|c|}{ Experiment 7} \\
\hline \multirow[t]{3}{*}{ Profiles } & slant & $F(3,6)=12.1$ & n.s. & n.s. & n.s. \\
\hline & tilt & n.s. & n.s. & $F(3,6)=20.6^{*}$ & n.s. \\
\hline & slant $\times$ tilt & n.s. & n.s. & $F(9,18)=3.4 \dagger$ & n.s. \\
\hline \multicolumn{6}{|c|}{ Experiment 8} \\
\hline \multirow[t]{3}{*}{ Two views } & slant & $F(3,6)=5.0 \dagger$ & n.s. & n.s. & n.s. \\
\hline & & $F(3,6)=22.6^{*}$ & n.s. & $F(3,6)=14.9^{*}$ & n.s. \\
\hline & slant $\times$ tilt & n.s. & n.s. & $F(9,18)=7.4^{*}$ & n.s. \\
\hline
\end{tabular}

Note - n.s, $=$ not significant at .05 level. *Significant at .01 level. †Significant at .05 level.

characteristic bias in the judgments of $z$ and $-z$, indicating that the alignment of the personal coordinate system with that of the measurement system was not perfect. This bias was similar to the result for judging the orientation of the real object. Although there is no ready explanation for such an effect, it would appear to be considerably less than the bias found in the SFM experiments. Thus, any biases in the measurement and display system is inadequate to explain the bias seen for the SFM displays.

In summary, the SFM results showed that although responded slant and tilt sometimes closely tracked what was being simulated, there were instances when subjects' responses contained a substantial amount of bias or variability. However, the variability was not so overwhelming as to totally mask an effect of increasing the simulated slant and tilt. The bias was most conspicuous in the slant judgments, particularly for low slants and when the tilt was $90^{\circ}$. One result worth noting was the large amount of slant bias found for the full rotations and two views at slants equal to $0^{\circ}$ and $90^{\circ}$. For these conditions, there were simple 2-D cues that should have provided the axis orientation, yet the subjects did not seem to use these cues effectively.

\section{GENERAL DISCUSSION}

The results of experiments with the real object demonstrated that the subjects were capable of accurately pointing the orientation of a static object over a wide range of directions. The other experiments, in which we investigated the accuracy of pointing the direction of the perceived axis of rotation for SFM displays, resulted in, relative to the real object, decreased performance for pointing the axis of rotation. However, for all the types of SFM displays, the subjects were sensitive to the simulated slants and tilts, and the bias in recovered slant and the variability in recovered tilt depended on the type of SFM display. The results for tilt were consistent with the theoretical predictions, and the instances of high bias and variability in tilt judgments were attributed to low signal-to-noise ratios, inherent when the axis approached the viewing direction. The results for slant cannot be entirely explained by any of the current models of recovery of structure from motion: it should not have been possible to recover slant for the experiments with two views, and there is no theoretical reason to predict the biases found with judgments of slant.

Given that the responses were not perfectly accurate, to interpret the results, it is helpful to try to partition the error into its possible sources. One possible source of error would be the visuomotor system, which must go from detecting an orientation to executing the appropriate motor commands (Flanders \& Soechting, 1993; Soechting \& Flanders, 1993). The intrinsic error of this system is most accurately reflected in the error found with the real object. For the real object, there were a few degrees of both bias and variability in the responses. Thus, since the error with SFM was much higher, it seems reasonable to conclude that the primary component of error in the SFM experiments arose from aspects of visual processing. However, associated with the SFM experiments,

Table 7

Results for Pointing the Cardinal Directions Averaged Across Subject and Experiment

\begin{tabular}{|c|c|c|c|c|c|c|}
\hline \multicolumn{3}{|c|}{ Direction } & \multicolumn{2}{|c|}{ Slant (deg) } & \multicolumn{2}{|c|}{ Tilt (deg) } \\
\hline$x$ & $y$ & $z$ & Bias & $S D$ & Bias & $S D$ \\
\hline 1.00 & 0.00 & 0.00 & -1.28 & 1.75 & -0.06 & 1.85 \\
\hline 0.00 & 1.00 & 0.00 & -0.27 & 1.74 & 0.27 & 1.23 \\
\hline 0.00 & 0.00 & 1.00 & 3.47 & 1.67 & nd & nd \\
\hline 0.00 & 0.00 & -1.00 & -4.43 & 1.52 & nd & nd \\
\hline
\end{tabular}

Note - nd $=$ tilt is not defined for this direction. 
there are several possible artifactual sources of the increase in error worth considering. These include the absence of an actual physical object with which to directly compare the finger position, the fact that between trials the same axis was simulated with different configurations, and possible competing flatness cues (unavoidable with the use of computer graphics displays). With the present data, it is impossible to distinguish among these possibilities, but it is important to keep in mind that the increase in bias and variability associated with going from the real object to SFM displays was probably not solely due to properties of the SFM process. With these considerations in mind, we will first compare the results against the theoretical predictions and then compare the performance between different types of SFM displays.

For the full rotations, there were various ways in which the direction of the axis of rotation could have been recovered. Thus, if we neglect the bias in slant judgments (which will be discussed later), we can see that the results were consistent with what would be theoretically predicted. In terms of performance, the variability in both the slant and the tilt was smallest for full rotations. The fact that variability was less for full rotations than for two views suggests that the subjects were able to integrate information over multiple views in order to obtain a more consistent estimate of the simulated motion.

For the profile displays, one technique to recover the axis of rotation would require a nearly complete rotation (Giblin et al., 1992; Pollick et al., 1991), and it relies on first recovering the tilt of the axis of rotation from the symmetry of the envelope of the profiles. Another technique uses special points on the profile, and it can recover the tilt of the axis of rotation from a short interval of rotation but cannot recover the slant unless the angular velocity is known (Giblin et al., in press). The results of Experiment 3 show at least one difficulty for the first method-that is, relative to performance for full rotations of dots, performance for profiles was substantially lowersuggesting that information available from the complete rotation, such as the envelope of the profiles, was not being used effectively. This and the fact that the profile results are similar to those for two views suggest that perhaps the second method is closer to modeling the type of method relevant for human perception.

For the two-view displays, it was theoretically impossible to completely recover the axis of rotation. Although several theories show that it is possible in principle to recover the tilt of the axis of rotation (Bennett et al., 1989; Koenderink \& Van Doorn, 1991; Todd \& Bressan, 1990), it is impossible to recover the slant uniquely. For example, the theory of Bennett et al. states that for every possible slant of the axis of rotation there is a valid 3-D interpretation; the theory of Todd and Bressan shows that the amount of slant is monotonically related to the amount of rotation (curl) extracted from the motion, but that the slope of this relation cannot be found. Thus, from these theories alone, it is impossible to explain why, for two views, slant responses increased in nearly the same way as simulated slant increased.
Although subjects' performance at two views does not entirely agree with current theoretical descriptions of shape recovery, it is similar to other psychophysical findings. For example, in a task involving shape judgments from two views, Liter, Braunstein, and Hoffman (in press) found that subjects, rather than spreading their responses over the entire range of possible responses outlined by Bennett et al. (1989), showed a tendency to report a shape similar to the one that generated the stimulus. In addition, Todd and Bressan (1990) have found that, given only two views of rotating line segments, subjects perform better at affine types of judgments; however, for 3-D metric judgments of line length and 3-D angle, they do perform better than chance. Taken together with the results of the present experiments, these results suggest that, from only two views, at least a rough 3-D metric representation is being formed.

If a 3-D metric interpretation (or indeed, any unique interpretation) is being formed from only two views, then there must be some additional regularity of the displays that is being exploited to obtain the interpretation. Many additional regularities of the stimuli could potentially be adequate to disambiguate the two views. Assumptions about spatial distribution, extent of rotation (angular velocity), and type of visual projection all might lead to successful heuristics for disambiguating a two-view stimulus. Of these assumptions, there is evidence that assuming small rotations between frames explains performance at the recovery of structure from motion (Proffitt, Rock, Hecht, \& Schubert, 1992). There is also evidence from multiple-view displays that planar motion allows perception of metric structure (Lappin \& Love, 1992).

Even if a metric representation is being formed, the current evidence suggests that it is not veridical in the sense of a Euclidean metric. The clearest evidence for this comes from the slant judgments. Judgments of slant increased as simulated slant increased; however, for low slants, subjects' responses deviated from veridical. This bias was most pronounced for full rotations and two views, where it was also related to the simulated tilt and became worse as tilt approached $90^{\circ}$. The increase in slant bias with decreasing simulated slant is surprising, because rotation about the line of sight should provide no cue to depth and these displays should have been seen as flat objects rotating about the line of sight. However, the present results suggest that for rotations about an axis near the line of sight, subjects (1) perceive some depth and (2) misperceive the axis of rotation. These two results are consistent with one another and suggest that subjects do not form a veridical percept for displays with large components of rotation about the line of sight.

The result that subjects' slant responses were not veridical for large components of rotation about the line of sight is also not consistent with the affine theories of SFM (Koenderink \& Van Doorn, 1991; Todd \& Bressan, 1990). These theories assume that, in order to obtain the affine structure, the component of rotation about the line of sight is accurately removed. If this component was being accurately removed, then the most consistent result would 
have been for slant judgments to become less biased (or at least, not increase in bias) as the component of rotation about the line of sight increased. However, this is not what we found; as the axis approached the viewing direction, variability remained constant while slant bias increased.

The breakdown in performance as the axis approaches the viewing direction has been commented on by Loomis and Eby (1989) and by Liter et al. (in press). They suggest that human vision uses heuristic processes, based on relative motion (removing curl), that provide only an approximate solution to the recovery of shape from motion, and that the approximation becomes less veridical as the axis approaches the viewing direction. If such an approximation exists, our findings suggest that it should be biased to overestimate slant and should be sensitive to the tilt as well as the slant of the axis of rotation.

Since the present results argue against either a strictly affine or a Euclidean metric representation, one can speculate on what sort of geometry is appropriate to model the recovery of structure from motion. One suggestion, which seems to embody a wide body of results, is that the human visual system is using a 3-D Euclidean metric system that simply does not work well. Thus, there could be an endless number of anisotropies (and strikingly low performances) exhibited by the system, but they would fall into no particular pattern and could not be completely described by any conventional geometry. An alternative to this would be an approach reminiscent of that of visual space research (Indow, 1991; Luneburg, 1947, 1950), where the failure of perception to fit a Euclidean metric led to modeling involving different geometries. Similar work in the domain of object recognition has recently been done by Pizlo (1993), who, after testing various of the traditional geometries and finding they failed to explain human performance, applied a geometry motivated by the constraints of image formation.

Yet another possible way to view the role of affine and Euclidean representations in the perception of SFM is to consider that the human visual system might be using elements of both. If we take, for example, a recent SFM algorithm proposed by Shapiro, Zisserman, and Brady (1993), we can see such a "hybrid" approach (see also Weinshall, 1993). Their algorithm uses successive two-frame computations to obtain optimal estimates of the affine parameters of motion. These affine parameters are then used pairwise (three frames; affine estimates from Frames 1 and 2 and Frames 2 and 3 ) to calculate the Euclidean distances and motion. Although such an approach certainly cannot account for all the present psychophysical results and is intended as a computer-vision algorithm, it demonstrates that in practice the distinction between affine and Euclidean representations might not be so clear-cut.

In conclusion, the present set of experiments showed that the subjects were able to orient the index finger in the direction of the axis of rotation for a variety of types of SFM displays. This ability was present for two-view displays with minimal amounts of motion and became less variable for full rotations when information could be in- tegrated over entire cycles of rotation. However, for profiles, the subjects did not seem able to use the information available in complete rotations. In addition, the subjects were able to recover the slant of the axis of rotation even for two views. These results are somewhat at odds with current formal theories of SFM and suggest that the subjects were using an additional assumption to recover the axis of rotation.

\section{REFERENCES}

Bennett, B., Hoffman, D., Nicola, J., \& Prakash, C. (1989). Structure from two orthographic views of rigid motion. Journal of the Optical Society of America, 6, 1052-1069.

Braunstein, M. L. (1962). Depth perception in rotating dot patterns: Effects of numerosity and perspective. Journal of Experimental Psychology, 64, 415-520.

Braunstein, M. L., Hoffman, D. D., \& Pollick, F. E. (1990). Discriminating rigid from nonrigid motion: Minimum points and views. Perception \& Psychophysics, 47, 205-214.

Cipolla, R., B Blake, A. (1992). Surface shape from the deformation of apparent contours. International Journal of Computer Vision, 9, 83-112.

CORTESE, J. M., \& ANDERSEN, G. J. (1991). Recovery of 3-D shape from deforming contours. Perception \& Psychophysics, 49, 315-327.

Dosher, B. A., LANDY, M. S., \& SPERLING, G. (1989). Ratings of kinetic depth in multidot displays. Journal of Experimental Psychology: Human Perception \& Performance, 15, 816-825.

EBY, D. W. (1992). The spatial and temporal characteristics of perceiving 3-D structure from motion. Perception \& Psychophysics, 51, 163-178.

Flanders, M., \& Soechting, J. F. (1993). Frames of reference for hand orientation. Manuscript submitted for publication.

Giblin, P. J., Pollick, F, E., \& Rycroft, J. E. (in press). Recovery of surfaces from profiles under circular motion with unknown axis. Journal of the Optical Society of America A.

Grblin, P. J., Rycroft, J. E., \& Pollick, F. E. (1994). Moving surfaces. In R. Fisher (Ed.), Design and applications of curves and surfaces: Mathematics of surfaces V (pp. 433-453). Oxford: Oxford University Press.

Giblin, P. J., \& Weiss, R. (1987). Reconstruction of surfaces from profiles. In Proceedings of the First International Conference of Computer Vision (pp. 136-144). Washington, DC: IEEE Computer Society Press.

GREEN, B. F., JR. (1961). Figure coherence in the kinetic depth effect. Journal of Experimental Psychology, 62, 272-282.

Hildreth, E. C., Grzywacz, N. M., Adelson, E. H., \& Inada, V. K. (1990). The perceptual buildup of three-dimensional structure from motion. Perception \& Psychophysics, 48, 19-36.

HuANG, T., \& LEE, C. (1989). Motion and structure from orthographic projections. IEEE Transactions on Pattern Analysis \& Machine Intelligence, 11, 536-540.

INDOw, T. (1991). A critical review of Luneburg's model with regard to global structure of visual space. Psychological Review, 98, 430-453.

KoEnderink, J. J. (1986). Optic flow. Vision Research, 26, 161-179.

Koenderink, J. J., \& V AN DooRn, A. J. (1991). Affine structure from motion. Journal of The Optical Society of America A, 8, 377-385.

LAPPIN, J. S., \& LovE, S. R. (1992). Planar motion permits perception of metric structure in stereopsis. Perception \& Psychophysics, 51, 86-102.

Liter, J. C., Braunstein, M. L., \& Hoffman, D. D. (in press). Inferring structure from motion in two-view and multi-view displays. Perception.

Loomis, J. M., \& EвY, D. W. (1988). Perceiving structure from motion: Failure of shape constancy. In Proceedings of the Second International Conference of Computer Vision (pp. 383-391). Washington, DC: IEEE Computer Society Press. 
Loомıs, J. M., \& EвY, D. W. (1989). Relative motion parallax and the perception of structure from motion. In Proceedings of the IEEE Workshop on Visual Motion (pp. 204-211). Washington, DC: IEEE Computer Society Press.

LUNEBURG, R. K. (1947). Mathematical analysis of binocular vision. Princeton, NJ: Princeton University Press.

LUNEBURG, R. K. (1950). The metric of binocular visual space. Journal of the Optical Society of America, 50, 637-642.

NoRman, J. F., \& TODD, J. T. (1993). The perceptual analysis of structure from motion for rotating objects undergoing affine stretching transformations. Perception \& Psychophysics, 53, 279-291.

Norman, J. F., \& TODD, J. T. (in press). The perception of rigid motion in depth from the optical deformations of shadows and occlusion boundaries. Journal of Experimental Psychology: Human Perception \& Performance.

PizLo, Z. (1993). Geometry of visual space. Investigative Ophthalmology \& Visual Science, 34, 708. (Abstract)

Pollick, F. E. (1993). The tradeoff between motion and structure: Rigid interpretations of affine stretching displays. Investigative Ophthalmology \& Visual Science, 34, 1034. (Abstract)

Pollick, F. E. (1994). Perceiving shape from profiles. Perception \& Psychophysics, 55, 152-161.

Pollick, F. E., \& CHENG, L. S. (1990). The effects of three-dimensional nonrigidity on discriminating rigid from nonrigid motion. Investigative Ophthalmology \& Visual Science, 31, 522. (Abstract)

Pollick, F. E., Giblin, P. J., Rycroft, J., \& Wilson, L. L. (1992). Human shape recovery from profiles. Behaviormetrika, 19, 65-79.

Proffit, D. R., Rock, I., Hecht, H., \& Schubert, J. (1992). Stereokinetic effect and its relation to the kinetic depth effect. Journal of Experimental Psychology: Human Perception \& Performance, 18, 3-21.

SAKATA, H., Kusunoki, M., \& TANAKA, Y. (in press). Neural mechanisms of perception of linear and rotary movement in depth in the parietal association cortex of the monkey.
Shapiro, L. S., Zisserman, A. P., \& Brady, M. (1993). Motion from point matches using affine epipolar geometry (Rep. No. OUEL 1994/93). Oxford: University of Oxford, Department of Engineering Robotics Research Group.

Soechting, J. F., \& Flanders, M. (1993). Parallel, interdependent channels for location and orientation in sensorimotor transformations for reaching and grasping. Journal of Neurophysiology, 70, 1137-1150.

ToDD, J. T. (1985). Perception of structure from motion: Is projective correspondence of moving elements a necessary condition? Journal of Experimental Psychology: Human Perception \& Performance, 11, 689-710.

Todd, J. T., Akerstrom, R. A., Reichel, F. D., \& Hayes, W. (1988). Apparent rotation in three-dimensional space: Effects of temporal, spatial, and structural factors. Perception \& Psychophysics, 43, 179-188.

ToDD, J. T., \& BREssAN, P. (1990). The perception of 3-dimensional affine structure from minimal apparent motion sequences. Perception \& Psychophysics, 48, 419-430.

UlLMAN, S. (1979). The interpretation of visual motion. Cambridge, MA: MIT Press.

Vaillant, R., \& Faugeras, O. D. (1992). Using extremal boundaries for 3-D object modeling. IEEE Transactions on Pattern Analysis \& Machine Intelligence, 14, 157-173.

Wallach, H., \& O'ConNELl, D. N. (1953). The kinetic depth effect. Journal of Experimental Psychology, 45, 205-217.

WeINSHALL, D. (1993). Model-based invariants for 3-D vision. International Journal of Computer Vision, 10, 27-42.

(Manuscript received October 13, 1992; revision accepted for publication December 28, 1993.) 